ESAIM: COCV 23 (2017) 721-749

DOI: $10.1051 / \mathrm{cocv} / 2016011$
ESAIM: Control, Optimisation and Calculus of Variations

www.esaim-cocv.org

\title{
A ONE-STEP OPTIMAL ENERGY DECAY FORMULA FOR INDIRECTLY NONLINEARLY DAMPED HYPERBOLIC SYSTEMS COUPLED BY VELOCITIES
}

\author{
Fatiha Alabau-Boussouira ${ }^{1}$, Zhiqiang WAng $^{2}$ And Lixin Yu ${ }^{3}$
}

\begin{abstract}
In this paper, we consider the energy decay of a damped hyperbolic system of wave-wave type which is coupled through the velocities. We are interested in the asymptotic properties of the solutions of this system in the case of indirect nonlinear damping, i.e. when only one equation is directly damped by a nonlinear damping. We prove that the total energy of the whole system decays as fast as the damped single equation. Moreover, we give a one-step general explicit decay formula for arbitrary nonlinearity. Our results shows that the damping properties are fully transferred from the damped equation to the undamped one by the coupling in velocities, different from the case of couplings through displacements as shown in [F. Alabau, C. R. Acad. Sci. Paris Sér. I Math. 328 (1999) 10151020; F. Alabau, P. Cannarsa and V. Komornik, J. Evol. Equ. 2 (2002) 127-150; F. Alabau, SIAM J. Control Optim. 41 (2002) 511-541; F. Alabau-Boussouira and M. Léautaud, ESAIM: COCV 18 (2012) 548-582] for the linear damping case, and in [F. Alabau-Boussouira, NoDEA 14 (2007) 643-669] for the nonlinear damping case. The proofs of our results are based on multiplier techniques, weighted nonlinear integral inequalities and the optimal-weight convexity method of [F. Alabau-Boussouira, Appl. Math. Optim. 51 (2005) 61-105; F. Alabau-Boussouira, J. Differ. Equ. 248 (2010) 1473-1517].
\end{abstract}

Mathematics Subject Classification. 35L05, 35Lxx, 93D15, 93D20.

Received March 17, 2015. Accepted February 18, 2016.

\section{INTRODUCTION}

Let $\Omega$ be a bounded subset of $\mathbb{R}^{n}$ with a smooth boundary denoted by $\Gamma$. We consider the following wave system

$$
\left\{\begin{array}{l}
u^{\prime \prime}-\Delta u+\alpha(x) v^{\prime}+\rho\left(x, u^{\prime}\right)=0 \quad \text { in } \Omega \times(0,+\infty), \\
v^{\prime \prime}-\Delta v-\alpha(x) u^{\prime}=0 \quad \text { in } \Omega \times(0,+\infty), \\
u=v=0 \quad \text { on } \Gamma \times(0,+\infty), \\
\left(u, u^{\prime}\right)(0)=\left(u^{0}, u^{1}\right),\left(v, v^{\prime}\right)(0)=\left(v^{0}, v^{1}\right) \quad \text { in } \Omega .
\end{array}\right.
$$

Keywords and phrases. Energy decay, nonlinear damping, wave equation, plate equation, weighted nonlinear integral inequality, optimal-weight convexity method.

1 IECL, Université de Lorraine and CNRS (UMR 7502), Délégation CNRS at LJLL UMR 7598, 57045 Metz, France. fatiha.alabau@univ-lorraine.fr

2 School of Mathematical Sciences and Shanghai Key Laboratory for Contemporary Applied Mathematics, Fudan University, Shanghai 200433, P.R. China. wzq@fudan.edu.cn

3 School of Mathematics and Information Sciences, Yantai University, Yantai 264005, P.R. China. fdylx01@sina.com.cn 
To a strong solution of this system, we associate the energy defined by

$$
E(t)=\frac{1}{2} \int_{\Omega}\left(\left|u^{\prime}\right|^{2}+|\nabla u|^{2}+\left|v^{\prime}\right|^{2}+|\nabla v|^{2}\right) \mathrm{d} x .
$$

One can show that the energy of the strong solutions of this system satisfies

$$
E^{\prime}(t)=-\int_{\Omega} u^{\prime} \rho\left(x, u^{\prime}\right) \mathrm{d} x
$$

In the sequel, the assumption on $\rho$ will ensure that $\rho(\cdot, s) s \geqslant 0$ a.e. in $\Omega$ and for all $s \in \mathbb{R}$, so that the energies of the strong solutions satisfy $E^{\prime}(t) \leq 0$. Hence the nonlinear term in our coupled system is indeed a damping term, so that one expects the energies to decay to 0 at infinity.

Let us recall that for the scalar damped wave equation, that is for

$$
u^{\prime \prime}-\Delta u+\rho\left(x, u^{\prime}\right)=0 \quad \text { in } \Omega \times(0,+\infty), u=0 \quad \text { on } \Gamma \times(0,+\infty) .
$$

It is well known that when the damping term is linear, i.e. when $\rho\left(., u^{\prime}\right)=a(\cdot) u^{\prime}$, where $a \geqslant 0$ a.e. on $\Omega$, the energy of the solution decays exponentially under some geometric conditions on the support of $a$ (see $[17,30]$ ). When the damping term $\rho(\cdot, \cdot)$ is nonlinear with respect to the second variable $[4,7,11]$, give, under some suitable geometric conditions, a one-step explicit energy decay formula in terms of the behavior of the nonlinear feedback close to the origin. These results rely on a general weighted nonlinear integral inequality together with an optimal-weight convexity method developed in [4]. If no geometric assumptions on the damping region are made, the decay is known to be of logarithmic type for a linear damping. Such types of results has first been proved in [28] (see also [18,22]).

Let us go back to the above wave-wave coupled system (1.1). At this stage, four main features characterize this system:

- only the first equation is damped

- the damping $\rho$ may have an arbitrary growth around 0 with respect to the second variable

- the coupling coefficient $\alpha$ may vanish in some parts of $\Omega$

- the coupling is acting through the velocities.

Let us now comment on these features.

- The fact that only one equation of the coupled system is damped refers to the so-called class of "indirect" stabilization problems initiated and studied in $[1,2,14]$ and further studied in $[5,12,15]$. Indeed, when dealing with coupled systems, it may be impossible or too expensive to damp each equation. Such an example is provided for instance by the Timoshenko system $[6,25,37]$. More generally, coupled systems involving some undamped equations, are said to be indirectly damped. From the point of view of applications in control theory, a challenging question is to determine whether the single feedback is sufficient to guarantee that the energy of the full system decays to 0 at infinity and to determine at which rate. In this latter case, the lack of feedback on the second equation is compensated by the coupling effects.

- Concerning the scalar wave equation. The case of general damping feedbacks, that is with arbitrary growth close to 0 , has received a lot of attention since more than a decade. The first result in this direction has been derived in [27], however no general simple explicit formula was provided except for linearly or polynomially growing dampings close to 0. Such first examples of explicit general formula are given in [33] (see also [32]), but this formula does not allow to recover in a single step the expected quasi-optimal energy decay rate in the polynomial case (or for polynomial-logarithmic growth). As far as we know, the first result giving a general one-step quasi-optimal semi-explicit formula is given in [4]. A further analysis based on a suitable and original classification of the feedback growth has been introduced in [7]. This classification gives a very simple one-step explicit energy decay formula for general feedbacks growth, provided that this growth is not close to a linear 
behavior. A more complex semi-explicit formula holds in the general case including feedbacks with a growth close to a linear one around 0 . By a one-step formula, we mean here, a formula which gives a decay rate depending explicitly on the feedback in a simple explicit way. In particular, this formula does not require further steps as in most of the existing literature to lead to explicit expressions. Moreover the optimality of the formula is proved for the corresponding finite dimensional systems or for semi-discretized scalar wave or plate equations, whereas optimality results are proved for some examples in the infinite dimensional case in [4], using results of [38,39].

For results using microlocal analysis and devoted to linearly damped semilinear wave equation, we refer the reader to [21,24] and the references therein. In [24], the authors prove the exponential stabilization of the linearly damped semilinear wave equation in the defocusing, subcritical case and under the Geometric Control Condition ( $G C C$ for short) of [17]). One can also see former results based on multiplier approach in for instance $[19,26,36,40,41]$, under less sharper geometric conditions.

- When the coupling coefficient is bounded below by a strictly positive constant, the coupling is active in the whole domain $\Omega$, so that the equations are coupled in the whole domain. If $\alpha$ is nonnegative on $\Omega$ but is allowed to vanish on a subset of $\Omega$, the equations are "uncoupled" in $\Omega \backslash \operatorname{supp}\{\alpha\}$, so that in this region the second equation is decoupled from the first equation and is undamped. Such cases are harder to handle. A first study in this framework, but for linearly damped wave-type equations coupled in displacements is given in [12].

- When indirect damping occurs through displacements, that is for systems coupled in displacements, as for instance

$$
\left\{\begin{array}{l}
u^{\prime \prime}-\Delta u+\alpha(x) v+a(x) u^{\prime}=0 \quad \text { in }(0, \infty) \times \Omega, \\
v^{\prime \prime}-\Delta v+\alpha(x) u=0 \quad \text { in }(0, \infty) \times \Omega, \\
u=v=0 \quad \text { on }(0, \infty) \times \Gamma, \\
\left(u(0), u^{\prime}(0)\right)=\left(u^{0}, u^{1}\right),\left(v(0), v^{\prime}(0)\right)=\left(v^{0}, v^{1}\right), \quad \text { in } \Omega,
\end{array}\right.
$$

it has been shown in [14] that even for constant coefficients $\alpha$, the energy of this linearly damped system never decays exponentially, but decays only polynomially with a decay rate depending on the smoothness of the initial data and a general lemma announced in [1] (see $[2,14]$ for a proof). These results are based on the method of higher order energies initiated in [1] and developed in [14] and [2] for the indirect boundary damping cases in an abstract setting and applied to various examples. The result of [14] has been generalized to the case that each coefficient $\alpha, a$ vanishes on a subset of $\Omega$ in [12,13], under certain assumptions on the supports of $\alpha$ and $a$ (roughly speaking they are both supposed to satisfy the $P M G C$ in [12] and the GCC in [13] (see Prop. 2.5)). Further results on coupled models with distributed dampings but satisfying hybrid boundary conditions have been obtained in [15]. Sharper results have been obtained through interpolation techniques extending the first results of [1]. Let us further mention that in [23], the author shows that the energy decays logarithmically under the assumption $\operatorname{supp}\{\alpha\} \cap \operatorname{supp}\{a\} \neq \emptyset$. Hence when the coupling acts through displacements, indirect stabilization occurs but in a weaker form than the one of the corresponding scalar case, since exponential stabilization does not hold even for a linearly damped case.

The loss of exponential stability was generally known to be a consequence of the loss of certain geometric conditions, namely $G C C$, in the case of direct and linear damping. The former results of [14] in the case of coupled systems in displacements show that the loss of exponential stability can appear for indirectly damped systems even if the damping is globally distributed (the support of the damping coefficient then satisfies the $G C C$ ) and the coupling coefficient is a constant. The present paper shows that the assumptions on the coupling operator are decisive, not only as far as the geometric assumptions on the support of its coefficient are concerned, but also as far as the order of the coupling operator is concerned. It shows, together with [14], an important new phenomenon in the loss or not of exponential stability. In particular, exponential stability occurs even in case of linear indirect damping, if the coupling acts in a stronger way (here through velocities). On the other hand, the results in [6,9] provide examples of systems of PDE's (namely Timoshenko beams), coupled through mixed zero and first order terms, globally indirectly damped and for which exponential stability may hold or not depending on the property of identical or distinct dynamics in the two coupled equations. Hence, this paper 
allows us to complete the literature on indirect damping properties for coupled systems, showing that positive results, strongly depend on the assumptions made on the dynamics, the order of the coupling and the geometric assumptions of the coupled systems.

The goal of this paper is to generalize the quasi-optimal energy decay formula given for scalar wave-type systems in [7] (see also [4]), to the case of coupled systems in velocities, under the above four features. More precisely, we prove, under some geometric conditions on the localized damping domain and the localized coupling domain, that the energy of these kinds of system decays as fast as that of the corresponding scalar nonlinearly damped equation. Hence, the coupling through velocities allows a full transmission of the damping effects, quite different from the coupling through the displacements.

The optimality of the above estimates has been proved for finite dimensional equations, including the semidiscretized wave equations in [7]. In the infinite dimensional setting, lower energy estimates or optimality are open questions. Optimality has been only proved in the particular case of one-dimensional wave equation with boundary damping (see $[4,38,39]$ ). Lower energy estimates have been established in [7-9] for scalar onedimensional wave equations, scalar Petrowsky equations in two-dimensions and Timoshenko systems. We use the comparison method developed in [9] to extend these results to one-dimensional wave systems coupled by velocities.

Remark 1.1. The method presented here is general and can easily be adapted to handle corresponding coupled systems of plate equations, elasticity models, and more complex examples in the spirit of the general approach given in [4]. Our aim through this paper is to give a general methodology on a concrete PDE example to show that if the damping effects are suitably transferred through the coupling operators, then indirect stabilization can produce damping mechanisms of the quality of a direct damping for the corresponding scalar equation.

Note that one can also present, with no additional mathematical originality and no gain with respect to applications, all our results by means of a "Lyapunov" type presentation. In this case, one introduces in a standard way a modified energy function $\mathcal{L}_{\varepsilon}=E+\varepsilon P$ where $E$ stands for the usual energy of the solution, and the terms in $P$ involve the successive required multipliers. This modified energy function is equivalent to the original energy for $\varepsilon$ sufficiently small. Then one writes a differential inequality for this modified energy, then uses a weakened form of the optimal-weight convexity method introduced in [4] (announced in [3]), replacing the original optimal weight by a less good weight function, and which gives weaker estimates. Mathematically and schematically, this consists to reproduce all the steps of the optimal-weight convexity integrating only with respect to space and replacing integral inequalities by differential inequalities for suitable functions as described above.

The rest of this paper is organized as follows: in Section 2, we give some basic preliminaries, assumptions and notations. The main results, including Theorems 3.1 on well-posedness of (1.1), Theorem 3.2 on energy decay for polynomially growing damping case, Theorem 3.4 on energy decay for general nonlinear damping case, Theorem 3.8 on lower energy estimates for one dimensional system (3.7), are presented in Section 3. Explicit decay rates corresponding to some typical dampings are also provided in Section 3.4. As the main tool of deriving the quasi-optimal one-step explicit energy decay formula, the optimal-weight convexity method together with general weighted nonlinear integral inequalities are introduced in Section 4. The proof of the main results, as well as the decay rates of Examples 3.11-3.14 are given in Section 5. Finally, Section 6 is used to prove Lemma 5.2 on weighted energy estimates for a single non homogeneous wave equation by the multiplier method.

\section{Preliminaries, ASSumptions And notations}

\section{- Notations}

For the simplicity of statement, we denote in the whole paper $L^{2}(\Omega)$ by $L^{2}, H_{0}^{1}(\Omega)$ by $H_{0}^{1}, H^{2}(\Omega)$ by $H^{2}$. Moreover, we say that the initial data are in the energy space whenever $\left(u^{0}, u^{1}\right) \in H_{0}^{1} \times L^{2}$ and $\left(v^{0}, v^{1}\right) \in H_{0}^{1} \times L^{2}$ and the initial data are smooth if $\left(u^{0}, u^{1}\right) \in\left(H^{2} \cap H_{0}^{1}\right) \times H_{0}^{1}$ and $\left(v^{0}, v^{1}\right) \in\left(H^{2} \cap H_{0}^{1}\right) \times H_{0}^{1}$. 


\section{- Geometric conditions}

As already mentioned in the introduction, stabilization results for wave-like systems require geometric conditions on the region where the feedback is active. In the sequel, we shall consider the so-called Piecewise Multipliers Geometric Condition (denoted by PMGC, in short), which has been first introduced in [31]:

Definition 2.1 (PMGC). We say that a subset $\omega \subset \Omega$ satisfies the PMGC, if there exist subsets $\Omega_{j} \subset \Omega$ having Lipschitz boundaries and points $x_{j} \in \mathbb{R}^{N}, j=1, \ldots, J$, such that $\Omega_{i} \cap \Omega_{j}=\emptyset$ for $i \neq j$ and $\omega$ contains a neighborhood in $\Omega$ of the set $\cup_{j=1}^{J} \gamma_{j}\left(x_{j}\right) \cup\left(\Omega \backslash \cup_{j=1}^{J} \Omega_{j}\right)$, where $\gamma_{j}\left(x_{j}\right)=\left\{x \in \partial \Omega_{j}:\left(x-x_{j}\right) \cdot \nu_{j}(x) \geqslant 0\right\}$ and $\nu_{j}$ is the outward unit normal to $\partial \Omega_{j}$.

\section{- Assumptions on the coupling coefficient}

We assume that the coupling function $\alpha \in C(\bar{\Omega})$ satisfies

$$
\left\{\begin{array}{l}
\exists \alpha_{+}>0, \alpha_{-}>0 \text { such that } \\
\alpha_{+} \geqslant \alpha(x) \geqslant 0, \quad \forall x \in \Omega, \\
\alpha(x) \geqslant \alpha_{-}>0, \quad \forall x \in \omega_{c} \subset \Omega,
\end{array}\right.
$$

where and $\omega_{c}$ is an open subset of $\Omega$ with positive measure.

\section{- Assumptions on the feedback}

We consider feedbacks $\rho$ with an arbitrary growth close to 0 . However, to give the reader a better insight of the scope and challenge of one-step explicit general quasi-optimal energy decay formulas, we first provide the result and proof for polynomially growing feedbacks, for which the proofs are easier and then the general result for arbitrary growing feedbacks. Hence, we detail below the two sets of assumptions: the one for the polynomial case, then those for the general case.

The assumptions in the case of polynomially growing feedbacks is as follows:

$$
\left\{\begin{array}{l}
\rho \in C(\bar{\Omega} \times \mathbb{R}), \rho(x, 0)=0 \quad \forall x \in \Omega, \\
s \mapsto \rho(x, s) \text { is nondecreasing } \forall x \in \Omega, \\
\exists c>0 \text { and } p \geqslant 1 \exists a \in C(\bar{\Omega}) \text { such that } \\
a(x)|s| \leqslant|\rho(x, s)| \leqslant c a(x)|s|, \quad \forall x \in \Omega,|s| \geqslant 1, \\
a(x)|s|^{p} \leqslant|\rho(x, s)| \leqslant c a(x)|s|^{\frac{1}{p}}, \quad \forall x \in \Omega,|s| \leqslant 1, \text { where } \\
a \geqslant 0 \text { on } \Omega, \exists a_{-}>0 \text { such that } a(x) \geqslant a_{-}, \quad \forall x \in \omega_{d} \subset \Omega,
\end{array}\right.
$$

where $\omega_{d}$ is an open subset of $\Omega$ with positive measure.

The assumptions in the case of arbitrary growing feedbacks is as follows

$$
\left\{\begin{array}{l}
\rho \in C(\bar{\Omega} \times \mathbb{R}), \rho(x, 0)=0 \quad \forall x \in \Omega, \\
s \mapsto \rho(x, s) \text { is nondecreasing } \forall x \in \Omega, \\
\exists c>0, \exists a \in C(\bar{\Omega}) \text { and } \exists g \in \mathcal{C}^{1}(\mathbb{R}) \text { such that } \\
a(x)|s| \leqslant|\rho(x, s)| \leqslant c a(x)|s|, \quad \forall x \in \Omega,|s| \geqslant 1, \\
a(x) g(|s|) \leqslant|\rho(x, s)| \leqslant c a(x) g^{-1}(|s|), \quad \forall x \in \Omega,|s| \leqslant 1, \text { where } \\
a \geqslant 0 \text { on } \Omega, \exists a_{-}>0 \text { such that } a(x) \geqslant a_{-}, \quad \forall x \in \omega_{d} \subset \Omega, \\
g \text { is a strictly increasing and odd function. }
\end{array}\right.
$$

Remark 2.2. Thanks to the hypotheses $\left(\mathrm{HF}_{p}\right)$ or $\left(\mathrm{HF}_{g}\right)$, we have

$$
\rho(x, s) s \geqslant 0, \quad \forall x \in \Omega, \forall s \in \mathbb{R},
$$

which ensures that the energy of the solutions of the above wave system is nonincreasing. 
Remark 2.3. Note that we can infer from $\left(\mathrm{HF}_{\mathrm{g}}\right)$ that for very $\varepsilon \in(0,1)$, there exists constants $c_{1}>0, c_{2}>0$ such that

$$
\left\{\begin{array}{l}
c_{1} a(x)|s| \leqslant|\rho(x, s)| \leqslant c_{2} a(x)|s|, \quad \forall x \in \Omega,|s| \geqslant \varepsilon, \\
c_{1} a(x) g(|s|) \leqslant|\rho(x, s)| \leqslant c_{2} a(x) g^{-1}(|s|), \quad \forall x \in \Omega,|s| \leqslant \varepsilon .
\end{array}\right.
$$

Remark 2.4. We assume that $\rho$ has a linear growth at infinity. When $\rho$ is bounded or has a nonlinear growth at infinity, the decaying properties of the energy change substantially, depending in particular on higher norms of the initial data even for linear dampings [35] (and the references therein).

Convexity assumptions on the feedback and some definitions

Assume that $\left(\mathrm{HF}_{\mathrm{g}}\right)$ holds. Then, following [4,7], we assume that the function $H$ defined by

$$
H(x)=\sqrt{x} g(\sqrt{x}),
$$

is strictly convex in a right neighborhood of 0 , i.e. on $\left[0, r_{0}^{2}\right]$ for some sufficiently small $r_{0} \in(0,1]$. We define the function $\widehat{H}$ on $\mathbb{R}$ by $\widehat{H}(x)=H(x)$ for every $x \in\left[0, r_{0}^{2}\right]$ and by $\widehat{H}(x)=+\infty$ otherwise, and we define the function $L$ on $[0,+\infty)$ by

$$
L(y)= \begin{cases}\frac{\widehat{H}^{\star}(y)}{y} & \text { if } y>0, \\ 0 & \text { if } y=0,\end{cases}
$$

where $\widehat{H}^{\star}$ is the convex conjugate function of $\widehat{H}$, defined by

$$
\widehat{H}^{\star}(y)=\sup _{x \in \mathbb{R}}\{x y-\widehat{H}(x)\} .
$$

By construction, the function $L:[0,+\infty) \rightarrow\left[0, r_{0}^{2}\right)$ is continuous, one-to-one, onto and increasing, moreover it is easy to check that

$$
0<L\left(H^{\prime}\left(r_{0}^{2}\right)\right)<r_{0}^{2}
$$

holds (see $[4,7]$ for a complete proof). We also define the function $\Lambda_{H}$ on $\left(0, r_{0}^{2}\right]$ by

$$
\Lambda_{H}(x)=\frac{H(x)}{x H^{\prime}(x)} .
$$

Finally, we define, for $x \geq 1 / H^{\prime}\left(r_{0}^{2}\right)$,

$$
\psi_{0}(x)=\frac{1}{H^{\prime}\left(r_{0}^{2}\right)}+\int_{1 / x}^{H^{\prime}\left(r_{0}^{2}\right)} \frac{1}{\theta^{2}\left(1-\Lambda_{H}\left(\left(H^{\prime}\right)^{-1}(\theta)\right)\right)} \mathrm{d} \theta .
$$

Then, $\psi_{0}(x) \geqslant x$ for all $x \in\left[\frac{1}{H^{\prime}\left(r_{0}^{2}\right)}, \infty\right)$. Moreover, $\psi_{0}$ is a strictly increasing function. Hence $\psi_{0}$ is a one-to-one and onto function from $\left[\frac{1}{H^{\prime}\left(r_{0}^{2}\right)}, \infty\right)$ on $\left[\frac{1}{H^{\prime}\left(r_{0}^{2}\right)}, \infty\right)$ (see also $[4,7]$ for the proof).

Remark 2.5. The function $\Lambda_{H}$ has been introduced for the first time by the first author in [7]. It is an essential tool to classify the feedback growths around 0 and to simplify the decay estimate formula given in [4] - without loosing optimality properties - for the feedbacks having a growth around 0 which is not close to a linear one (as explained below).

Remark 2.6. Note that due to our convexity assumptions, we have

$$
\Lambda_{H}([0,1]) \subset[0,1] .
$$

When $g(x)=x$ (linear feedback case), $\Lambda_{H}(.) \equiv 1$. If we now set for instance, $g(x)=x(\ln (1 / x))^{-q}$ in $(0, \varepsilon]$ with $q>0$ and $\varepsilon>0$, then $\limsup _{x \rightarrow 0^{+}} \Lambda_{H}(x)=1$. Many other examples of feedbacks such that $\limsup _{x \rightarrow 0^{+}} \Lambda_{H}(x)=1$ can 
be given, they characterize a growth which is close to a linear one. This leads to the following definition:

Definition 2.7. We say that a feedback $\rho$ satisfying $\left(\mathrm{HF}_{g}\right)$ has a growth close to a linear one in a neighborhood of 0 , if it is such that the function $H$ defined by $(2.3)$ satisfies $\lim \sup \Lambda_{H}(x)=1$. Otherwise, one says that the feedback $\rho$ is away from a linear growth.

On the opposite side, for functions $g$ which converge very fast to 0 as $x$ goes to 0 , such as for instance $g(x)=\mathrm{e}^{-1 / x}$ for $x \in(0, \varepsilon]$ (and many other examples), one has $\lim \sup \Lambda_{H}(x)=0$.

For polynomially growing feedbacks, e.g. when $g(x)=x^{p}$ with $p>1$, we have $\Lambda_{H}(.) \equiv \frac{2}{p+1}$. For feedbacks such as $g(x)=x^{p}(\ln 1 / x)^{q}$ with $p>1, q>0$, we still have $\limsup _{x \rightarrow 0^{+}} \Lambda_{H}(x)=\frac{2}{p+1}$.

We will see later on, in Theorem 3.4, that the case of feedbacks close to a linear behavior as $x$ goes to 0 has to be distinguished from the other cases.

Remark 2.8. Note that when $g^{\prime}(0) \neq 0, g$ has a linear growth close to 0 . Therefore, this case is similar to the linear case which is already well-known. We thus focus in the sequel on the cases where $g^{\prime}(0)=0$.

\section{MAIN RESUlts}

\subsection{Well-posedness}

We set $\mathcal{H}=\left(H_{0}^{1} \times L^{2}\right)^{2}$ and set $U=(u, p, v, q)$. We equip $\mathcal{H}$ with the scalar product

$$
\langle U, \widetilde{U}\rangle=\int_{\Omega}(\nabla u \cdot \nabla \widetilde{u}+p \widetilde{p}+\nabla v \cdot \nabla \widetilde{v}+q \widetilde{q}) \mathrm{d} x .
$$

Theorem 3.1. Assume $\left(\mathrm{HF}_{g}\right)$ and that $\alpha \in L^{\infty}(\Omega)$. Then for all initial data in energy space, there exists a unique solution $(u, v) \in \mathcal{C}\left([0,+\infty) ;\left(H_{0}^{1}\right)^{2}\right) \cap \mathcal{C}^{1}\left([0,+\infty) ;\left(L^{2}\right)^{2}\right)$ of $(1.1)$. Moreover for any smooth initial data, the solution satisfies $(u, v) \in L^{\infty}\left([0,+\infty) ;\left(H^{2} \cap H_{0}^{1}\right)^{2}\right) \cap W^{1, \infty}\left([0,+\infty) ;\left(H_{0}^{1}\right)^{2}\right) \cap W^{2, \infty}\left([0,+\infty) ;\left(L^{2}\right)^{2}\right)$. Moreover, in this latter case the energy of order one, defined by

$$
E_{1}(t)=\frac{1}{2} \int_{\Omega}\left(\left|u_{t t}\right|^{2}+\left|\nabla u_{t}\right|^{2}+\left|v_{t t}\right|^{2}+\left|\nabla v_{t}\right|^{2}\right) \mathrm{d} x
$$

is non increasing, i.e.,

$$
E_{1}(t) \leq E_{1}(0)
$$

\subsection{One-step quasi-optimal energy decay rate for the wave-wave system}

\section{First case: Polynomially growing dampings close 0}

For the sake of clarity, we first provide the results in the case of a polynomially growing feedback $s \mapsto \rho(\cdot, s)$.

Theorem 3.2. Assume that $p>1,\left(\mathrm{HF}_{p}\right)$ and $(\mathrm{HC})$ hold. Assume also that $\omega_{d}$ and $\omega_{c}$ satisfy the PMGC. Then there exists $\alpha^{*}>0$ such that for any $\alpha_{+} \in\left(0, \alpha^{*}\right]$ and any non vanishing initial data in the energy space, the total energy of (1.1) defined in (1.2) decays as

$$
E(t) \leqslant C_{E(0)} t^{\frac{2}{1-p}}, \quad \forall t \in\left[T_{E(0)},+\infty\right),
$$

where $C_{E(0)}, T_{E(0)}>0$ are constants depending on $E(0)$.

Remark 3.3. If $p=1$, we can follow the Proof of Theorem 3.2 and obtain, by using Lemma 4.6 instead of Lemma 4.3 , the exponential stability for system (1.1)

$$
E(t) \leqslant C E(0) \mathrm{e}^{-\kappa t}, \quad \forall t \in[0,+\infty),
$$

where $C>0, \kappa>0$ are constants independent of the initial data. 


\section{Second case: Arbitrarily growing dampings close to the origin}

If we consider a more general nonlinear damping $\rho$, we provide below a quasi-optimal one-step explicit energy decay formula following the optimal-weight convexity method together with general weighted nonlinear integral inequalities developed in $[4,7]$.

Theorem 3.4. Assume $\left(\mathrm{HF}_{g}\right)$ and $(\mathrm{HC})$ hold. Assume that the function $H$ defined by (2.3) is strictly convex in $\left[0, r_{0}^{2}\right]$ for some sufficiently small $r_{0} \in(0,1]$ and satisfies $H(0)=H^{\prime}(0)=0$. We define the maps $L$ and $\Lambda_{H}$ respectively as in (2.4) and (2.6). Assume also that $\omega_{d}$ and $\omega_{c}$ satisfy the PMGC. Then there exists $\alpha^{*}>0$ such that for any $\alpha_{+} \in\left(0, \alpha^{*}\right]$ and any non vanishing initial data in the energy space, the total energy of (1.1) defined in (1.2) decays as

$$
E(t) \leq 2 \beta_{E(0)} L\left(\frac{1}{\psi_{0}^{-1}\left(\frac{t}{M}\right)}\right), \quad \forall t \geq \frac{M}{H^{\prime}\left(r_{0}^{2}\right)},
$$

where $\beta_{E(0)}$ is defined by (5.30), $M$ is defined by (5.34) and independent of E(0). Furthermore, if $\limsup \Lambda_{H}(x)<1$, then $E$ satisfies the following simplified decay rate $x \rightarrow 0^{+}$

$$
E(t) \leq 2 \beta_{E(0)}\left(H^{\prime}\right)^{-1}\left(\frac{\kappa M}{t}\right), E(t) \leq 2 \beta_{E(0)}\left(H^{\prime}\right)^{-1}\left(\frac{\kappa M}{t}\right)
$$

for $t$ sufficiently large, and where $\kappa>0$ is a constant independent of $E(0)$.

Remark 3.5. In both Theorems 3.2 and 3.4, we assume that the support of the coupling and of the damping coefficents satisfy the $P M G C$. In the nonlinear damping case, as far as we know, there exists no direct proof of a similar result under the $G C C$, even for single scalar wave equation. Instead, it is possible to prove such a result by an indirect method as in [11] (see also [16] in a more general setting). It consists on deducing the energy decay rate for the nonlinearly damped scalar wave equation from an observability result for the conservative scalar equation. Then one can use the classical results of [17] which establishes the observability inequality for the conservative scalar wave equation under the GCC. As explained in [11], this indirect approach allows to optimize both the geometric condition on the support of the damping and the decay rate of the energy in the scalar case. We plan to perform such an analysis in a future work for the indirectly damped wave systems considered here. Note also, that smoothness assumptions on the domain and on the damping coefficient are less restrictive in the direct method.

Remark 3.6. Our results in Theorems 3.2 and 3.4 are valid under a smallness assumption on the coupling coefficient. It allows us to deal with a positive total energy $E$ (which includes the coupling effects) and also to absorb coupling terms at crucial steps of our proofs, but it is not always necessary. Actually, inspired by the present article, the asymptotic stability of wave equations coupled by velocities with general constant coefficients have been studied in [20]. The exponential stability results are determined and classified by the algebraic property of the coupling and damping coefficients. In particular, if $\alpha(x) \equiv \alpha$ and $\rho\left(x, u_{t}\right) \equiv a u_{t}$ in $\Omega$ with $a>0$, then the coupled system is always exponentially stable.

Remark 3.7. Note that when $g(x)=x^{1 / p}$ wih $p>1$, then $H(x)=x^{(p+1) / 2}$ so that $\limsup _{x \rightarrow 0^{+}} \Lambda_{H}(x)=\frac{2}{p+1}<1$. Then the formula (3.6) gives back the energy decay rate of $t^{-2 /(p-1)}$ given in Theorem 3.2.

\subsection{Lower energy estimates}

The optimality of the above estimates are open questions. Here we use the comparison method developed in [9] to establish the lower estimate of the energy of the one-dimensional coupled wave system. Actually, we consider 
the following wave-wave system in $\Omega=(0,1) \subset \mathbb{R}$ and $\rho(x, s)=a(x) g(s)$ for all $x \in \Omega$ and all $s \in \mathbb{R}$.

$$
\left\{\begin{array}{l}
u_{t t}-u_{x x}+\alpha(x) v_{t}+a(x) g\left(u_{t}\right)=0, \quad \text { in } \Omega \times(0,+\infty), \\
v_{t t}-v_{x x}-\alpha(x) u_{t}=0, \quad \text { in } \Omega \times(0,+\infty), \\
u(t, 0)=u(t, 1)=v(t, 0)=v(t, 1)=0, \quad \text { for } t \in(0,+\infty), \\
\left(u, u_{t}\right)(0, x)=\left(u^{0}(x), u^{1}(x)\right),\left(v, v_{t}\right)(0, x)=\left(v^{0}(x), v^{1}(x)\right), \quad \text { for } x \in \Omega .
\end{array}\right.
$$

We define $H$ by (2.3), $\Lambda_{H}$ by (2.6) and consider the following assumptions

$$
\left\{\begin{array}{l}
\exists r_{0}>0 \text { such that the function } H:\left[0, r_{0}^{2}\right] \mapsto \mathbb{R} \text { defined by }(2.3) \\
\text { is strictly convex on }\left[0, r_{0}^{2}\right] \text { and } \\
\text { either } 0<\liminf _{x \rightarrow 0+} \Lambda_{H}(x) \leq \limsup _{x \rightarrow 0+} \Lambda_{H}(x)<1 \\
\text { or there exist } \mu>0 \text { and } z_{1} \in\left(0, z_{0}\right] \text { with }(5.39) \text { such that } \\
\quad 0<\liminf _{x \rightarrow 0+}\left(\frac{H(\mu x)}{\mu x} \int_{x}^{z_{1}} \frac{1}{H(y)} d y\right) \text { and } \limsup _{x \rightarrow 0+} \Lambda_{H}(x)<1 .
\end{array}\right.
$$

Theorem 3.8. Assume that $\left(\mathrm{HF}_{g}\right),\left(\mathrm{HF}_{l}\right)$, and $(\mathrm{HC})$ hold. Assume also that $\omega_{d}$ and $\omega_{c}$ satisfy the PMGC, and that $\alpha_{+} \in\left(0, \alpha^{*}\right]$ where $\alpha^{*}>0$ is as in Theorem 3.4. Then for all non vanishing smooth initial data, there exist $T_{0}>0$ and $T_{1}>0$ such that the energy of (3.7) satisfies the lower estimate

$$
E(t) \geq \frac{1}{\gamma_{s}^{2} C_{s}^{2}}\left(\left(H^{\prime}\right)^{-1}\left(\frac{1}{t-T_{0}}\right)\right)^{2}, \quad \forall t \geq T_{1}+T_{0},
$$

where $\gamma_{s}=4 \sqrt{E_{1}(0)}, C_{s}$ is as in Lemma 5.7 .

Remark 3.9. In [38,39], optimality of the upper estimates have been shown to hold for the one-dimensional scalar wave equation (or in radial cases) with a nonlinear damping acting on the boundary for particular sets of initial data. It woud be very interesting to extend this analysis to the coupled systems we consider here, and in particular to check that at least for particular initial data the solutions decay exactly at the speed given by the upper estimate.

Remark 3.10. Similar lower energy estimates have been established for a different example of coupled system in [9], namely the Timoshenko beams. This system is a one-dimensional model coupling two wave equations through mixed zero and first order coupling operators in space. The proof is also based on an energy comparison principle together with interpolation type arguments.

\subsection{Some examples of decay rates}

For the sake of completeness, we give some significative examples (taken from $[4,7]$ ) of feedback growths together with the resulting energy decay rate when applying our results. In the sequel, $C_{E(0)}>0$ stands for a constant depending on $E(0)$, while $C_{E_{1}(0)}^{\prime}>0$ is a constant depending on $E_{1}(0)$.

Example 3.11 (The polynomial case). Let $g(x)=x^{p}$ on $\left(0, r_{0}\right]$ with $p>1$. Then the energy of (1.1) satisfies the estimate

$$
E(t) \leq C_{E(0)} t^{-\frac{2}{p-1}}
$$

for $t$ sufficiently large and for all non vanishing initial data in the energy space. Moreover, the energy of (3.7) satisfies the estimate

$$
E(t) \geqslant C_{E_{1}(0)}^{\prime} t^{-\frac{4}{p-1}}
$$

for $t$ sufficiently large and for all non vanishing smooth initial data. 
Example 3.12 (Exponential growth of the feedback). Let $g(x)=\mathrm{e}^{-\frac{1}{x^{2}}}$ on $\left(0, r_{0}\right]$. Then the energy of $(1.1)$ decays as

$$
E(t) \leq C_{E(0)}(\ln (t))^{-1},
$$

for $t$ sufficiently large and for all non vanishing initial data in the energy space. Moreover, the energy of (3.7) satisfies the estimate

$$
E(t) \geqslant C_{E_{1}(0)}^{\prime}(\ln (t))^{-2},
$$

for $t$ sufficiently large and for all non vanishing smooth initial data.

Example 3.13 (Polynomial-logarithmic growth). Let $g(x)=x^{p}(\ln (1 / x))^{q}$ on $\left(0, r_{0}\right]$ with $p>2$ and $q>1$. Then the energy of (1.1) decays as

$$
E(t) \leq C_{E(0)} t^{-2 /(p-1)}(\ln (t))^{-2 q /(p-1)}
$$

for $t$ sufficiently large and for all non vanishing initial data in the energy space. Moreover the energy of (3.7) satisfies the estimate

$$
E(t) \geqslant C_{E_{1}(0)}^{\prime} t^{-4 /(p-1)}(\ln (t))^{-4 q /(p-1)},
$$

for $t$ sufficiently large and for all non vanishing smooth initial data.

Example 3.14 (Faster than Polynomials, Slower than Exponential). Let $g(x)=\mathrm{e}^{-(\ln (1 / x))^{p}}$ on $\left(0, r_{0}\right]$ with $p>2$. Then the energy of (1.1) decays as

$$
E(t) \leq C_{E(0)} \mathrm{e}^{-2(\ln (t))^{1 / p}},
$$

for $t$ sufficiently large and for all non vanishing initial data in the energy space. Moreover the energy of (3.7) satisfies the estimate

$$
E(t) \geqslant C_{E_{1}(0)}^{\prime} \mathrm{e}^{-4(\ln (t))^{1 / p}},
$$

for $t$ sufficiently large and for all non vanishing smooth initial data.

Remark 3.15. For these above four examples, one can show that $\lim _{x \rightarrow 0^{+}} \Lambda_{H}(x)<1$. Moreover, it is proved in [7], that the above decay rates are optimal in the finite dimensional case.

\section{Weighted NONLINEAR INTEGRAL INEQUALITIES AND DECAY RATES}

Definition 4.1. We say that a nonnegative function $E$ defined on $[0,+\infty)$ satisfies a weighted nonlinear integral inequality if there exists a nonnegative function $w$ defined on $[0, \eta)$ with $0<\eta \leq+\infty$ and a constant $M>0$ such that $E([0,+\infty)) \subset[0, \eta)$ and

$$
\int_{t}^{\infty} w(E(s)) E(s) \mathrm{d} s \leq M E(t), \quad \forall t \in[0,+\infty) .
$$

Definition 4.2. We say that such a weight function $w$ is of "polynomial" type, if there exists $\alpha>0$ such that

$$
w(s)=s^{\alpha}, \quad \forall s \geqslant 0, \text { with } \eta=+\infty .
$$

It is well-known that when $E$ is a nonnegative, nonincreasing absolutely continuous function satisfying (4.1) with a polynomial weight function, then $E$ satisfies an optimal decay rate at infinity, proved in ([26], Thm. 9.1) (see also herein for other references), that we recall in the next subsection. 


\subsection{Polynomial weights}

Lemma 4.3 ([26], Thm. 9.1). Assume that $E:[0,+\infty) \mapsto[0,+\infty)$ is a non-increasing function and that there are two constants $\alpha>0$ and $T>0$ such that

$$
\int_{t}^{\infty} E^{\alpha+1}(s) \mathrm{d} s \leq T E(0)^{\alpha} E(t), \quad \forall t \in[0,+\infty) .
$$

Then we have

$$
E(t) \leq E(0)\left(\frac{T+\alpha t}{T+\alpha T}\right)^{-\frac{1}{\alpha}}, \quad \forall t \in[T,+\infty) .
$$

Remark 4.4. Note that when applied to decay estimates for dissipative systems, the above Lemma has to be used with a constant $T$ which blows up as $E(0)=0$, so that the minimal time for which the above decay estimate is valid, blows up as $E(0)=0$. This result can be reformulated as below, to give an estimate which is valid for $E(0)>0$ as well as for $E(0)=0$ and for any $t \geqslant 0$ as explained below.

Corollary 4.5. Assume that $E:[0,+\infty) \mapsto[0,+\infty)$ is a non-increasing function and that there are two constants $\alpha>0$ and $M>0$ such that

$$
\int_{t}^{\infty} E^{\alpha+1}(s) \mathrm{d} s \leq M E(t), \quad \forall t \in[0,+\infty) .
$$

Then we have

$$
E(t) \leq E(0) \min \left(\left(\frac{M(\alpha+1)}{M+\alpha E(0)^{\alpha} t}\right)^{1 / \alpha}, 1\right), \quad \forall t \geqslant 0 .
$$

In particular for $E(0)>0$, we deduce that

$$
E(t) \leq E(0)\left(\frac{M(\alpha+1)}{M+\alpha E(0)^{\alpha} t}\right)^{1 / \alpha}, \quad \forall t \geqslant M E(0)^{-1 / \alpha} .
$$

Lemma 4.6 ([26], Thm. 8.1). Assume that $E:[0,+\infty) \mapsto[0,+\infty)$ is a non-increasing function and that there is a constant $T>0$ such that

$$
\int_{t}^{\infty} E(s) \mathrm{d} s \leq T E(t), \quad \forall t \in[0,+\infty) .
$$

Then we have

$$
E(t) \leq E(0) \mathrm{e}^{1-t / T}, \quad \forall t \in[T,+\infty) .
$$

\subsection{General weights}

For general weight functions, semi-explicit optimal decay rates have been derived for the first time in [4], and later on a simplified form of the rates in [7].

Let $\eta>0$ and $M>0$ be fixed and $w$ be a strictly increasing function from $[0, \eta)$ onto $[0,+\infty)$. For any $r \in(0, \eta)$, we define a function $K_{r}$ from $(0, r]$ on $[0,+\infty)$ by:

$$
K_{r}(\tau)=\int_{\tau}^{r} \frac{\mathrm{d} y}{y w(y)}
$$

and a function $\psi_{r}$ which is a strictly increasing onto function defined from $\left[\frac{1}{w(r)},+\infty\right)$ on $\left[\frac{1}{w(r)},+\infty\right)$ by:

$$
\psi_{r}(z)=z+K_{r}\left(w^{-1}\left(\frac{1}{z}\right)\right) \geq z \quad \forall z \geq \frac{1}{w(r)}
$$


We can now formulate our weighted integral inequality:

Theorem 4.7 ([4], Thm. 2.1). We assume that $E$ is a nonincreasing, absolutely continuous function from $[0,+\infty)$ on $[0, \eta)$, satisfying the inequality

$$
\int_{S}^{T} w(E(t)) E(t) \mathrm{d} t \leq M E(S), \quad \forall 0 \leq S \leq T .
$$

Then E satisfies the following estimate:

$$
E(t) \leq w^{-1}\left(\frac{1}{\psi_{r}^{-1}\left(\frac{t}{M}\right)}\right) \quad \forall t \geq \frac{M}{w(r)},
$$

where $r>0$ is such that

$$
\frac{1}{M} \int_{0}^{+\infty} E(\tau) w(E(\tau)) \mathrm{d} \tau \leq r \leq \eta
$$

In particular, we have $\lim _{t \rightarrow+\infty} E(t)=0$ with the decay rate given by (4.5).

Theorem 4.8 ([7], Thm. 2.3). Let $H$ be a strictly convex function on $\left[0, r_{0}^{2}\right]$ such the $H(0)=H^{\prime}(0)=0$. We define $L$ and $\Lambda_{H}$ as above. Let $E$ be a given nonincreasing, absolutely continuous function from $[0,+\infty)$ on $[0,+\infty)$ with $E(0)>0, M>0$ and $\beta$ is a given parameter such that

$$
\frac{E(0)}{2 L\left(H^{\prime}\left(r_{0}^{2}\right)\right)} \leq \beta .
$$

In addition, E satisfies the following weighted nonlinear inequality

$$
\int_{S}^{T} L^{-1}\left(\frac{E(t)}{2 \beta}\right) E(t) \mathrm{d} t \leq M E(S), \quad \forall 0 \leq S \leq T .
$$

Then E satisfies the following estimate:

$$
E(t) \leq 2 \beta L\left(\frac{1}{\psi_{0}^{-1}\left(\frac{t}{M}\right)}\right) \quad \forall t \geq \frac{M}{H^{\prime}\left(r_{0}^{2}\right)} .
$$

Furthermore, if $\limsup _{x \rightarrow 0^{+}} \Lambda_{H}(x)<1$, then $E$ satisfies the following simplified decay rate

$$
E(t) \leq 2 \beta\left(H^{\prime}\right)^{-1}\left(\frac{\kappa M}{t}\right)
$$

for $t$ sufficiently large, and where $\kappa>0$ is a constant independent of $E(0)$.

\section{Proof of MAin Results}

In this section, we prove the main results including Theorems 3.1-3.8 and the decay rates in Examples 3.113.14 .

\subsection{Proof of Theorem 3.1}

We define the following unbounded nonlinear operator $\mathcal{A}$ in $\mathcal{H}$ by

$$
\mathcal{A} U=(p, \Delta u-\alpha q-\rho(., p), q, \Delta v+\alpha p),
$$


with the domain

$$
D(\mathcal{A})=\{U \in \mathcal{H} ; \mathcal{A} U \in \mathcal{H}\} .
$$

It is easy to check that $\left.D(\mathcal{A})=H^{2} \cap\left(H_{0}^{1}\right) \times H_{0}^{1}\right)^{2}$. Moreover, since $\rho$ is nondecreasing with respect the second variable, we have for all $U, \tilde{U} \in D(\mathcal{A})$,

$$
\langle\mathcal{A} U-\mathcal{A} \widetilde{U}, U-\widetilde{U}\rangle=-\int_{\Omega}(\rho(x, p)-\rho(x, \widetilde{p}))(p-\widetilde{p}) \mathrm{d} x \leq 0,
$$

Thus $-\mathcal{A}$ is a monotone operator. We now claim that $-\mathcal{A}$ is a maximal operator. We proceed as follows. We denote by $A$ the unbounded operator in $L^{2}$ defined by $A=-\Delta$ and $D(A)=H^{2} \cap H_{0}^{1}$. Then $I-A$ is invertible as an operator acting from $H_{0}^{1}$ in $H^{-1}(\Omega)$, so that the operator $(I-A)^{-1}$ is a well-defined, self-adjoint and if $w \in L^{2}$ then $(I-A)^{-1} w \in H^{2} \cap H_{0}^{1}$. Then for any $F=(f, g, h, r) \in \mathcal{H}$, the equation

$$
(I-\mathcal{A}) U=F
$$

with $U=(u, p, v, q) \in D(\mathcal{A})$ is equivalent to

$$
\left\{\begin{array}{l}
u-\Delta u+\alpha(I-A)^{-1}(\alpha u)+\rho(., u-f)=G, \\
v=(I-A)^{-1}\left(H_{2}+\alpha u\right), \\
p=u-f, q=v-h,
\end{array}\right.
$$

where

$$
\left\{\begin{array}{l}
H_{1}=g+f+\alpha h \in L^{2}, \\
H_{2}=r+h-\alpha f \in L^{2}, \\
G=H_{1}-\alpha(I-A)^{-1} H_{2} \in L^{2} .
\end{array}\right.
$$

We define for $\theta \in \mathbb{R}$

$$
R(x, \theta)=\int_{0}^{\theta} \rho(x, s) \mathrm{d} s .
$$

Let us define the functional $J: H_{0}^{1} \mapsto \mathbb{R}$ defined by

$$
J(u)=\int_{\Omega}\left(\frac{1}{2}\left(u^{2}+|\nabla u|^{2}+\left|(I-A)^{-1 / 2}(\alpha u)\right|^{2}\right)+R(x, u-f)-G u\right) \mathrm{d} x .
$$

Note that thanks to our hypotheses, $|\rho(x, s)| \leq C(1+|s|)$ for all $(x, s) \in \Omega \times \mathbb{R}$, so that $J$ is well-defined and continuously differentiable on $H_{0}^{1}$. Moreover, we have

$$
J^{\prime}(u) \cdot \varphi=\int_{\Omega}\left(u \varphi+\nabla u \cdot \nabla \varphi+(I-A)^{-1 / 2}(\alpha u)(I-A)^{-1 / 2}(\alpha \varphi)+\rho(x, u-f) \varphi-G \varphi\right) \mathrm{d} x .
$$

We denote by $\|\cdot\|$ the $L^{2}$ norm. Since $\rho$ is nondecreasing with respect to the second variable, $J$ is a convex function and we also have

$$
J(u) \geqslant\left(\frac{\|u\|}{2}-\|G\|\right)\|u\|+\frac{\|\nabla u\|^{2}}{2}
$$

so that $J(u) \longrightarrow+\infty$ as $\|\nabla u\| \longrightarrow+\infty$. Hence $J$ is coercive. Therefore $J$ attains a minimum at some point $u \in H_{0}^{1}$, which satisfies the Euler equation

$$
J^{\prime}(u)=0
$$


The usual elliptic theory implies that the weak solution $u$ of the variational problem

$$
\left\{\begin{array}{l}
u \in H_{0}^{1}, \\
J^{\prime}(u) \cdot \varphi=0, \quad \forall \varphi \in H_{0}^{1}
\end{array}\right.
$$

is in $H^{2}$. Hence $u \in H^{2} \cap H_{0}^{1}$. By defining $v$ as in (5.1), and $p, q$ as in (5.1), it follows that $U=(u, p, v, q) \in D(\mathcal{A})$ and $(I-\mathcal{A}) U=F$. Hence $-\mathcal{A}$ is a maximal monotone operator. We conclude Theorem 3.1 using the classical theory of maximal monotone operator (see e.g. [26] and the references therein).

\subsection{Proof of Theorems 3.2 and 3.4}

The proof will be divided in three steps, following those described in [10] (see also $[4,7]$ ).

- Step 1: we first prove that the energy $E$ satisfies a suitable dominant energy estimate. This is the step in which the geometric assumptions $P M G C$ on both the damping and the coupling regions are used, together with suitable multipliers adapted to the coupled structure of the wave-wave system. The proof is valid without specifying the growth assumptions on the feedback $\rho$.

- Step 2: we then prove that nonnegative and nonincreasing functions $E$ satisfying a suitable dominant energy estimate, satisfies a general weighted nonlinear inequality. In the case of polynomially growing feedbacks $\rho$, the proof is easier since the weight function for integral inequalities is known. The general growing case relies on the optimality-convexity method of the first author [4].

- Step 3: we deduce energy decay rates, applying Corollary 4.5 for polynomially growing feedbacks, whereas applying Theorem 4.8 for general growing feedbacks.

Let us start with Step 1. We use the dominant energy method as developed and explained by the first author in $[4,10]$. This method consists in estimating time integrals of the nonlinear weighted energy of the system by corresponding dominant weighted energies, here in the frictional case, it means by respectively the nonlinear kinetic energy and the localized linear kinetic energy. Note that this step is valid for feedbacks with polynomial as well as arbitrary growth close to the origin.

Theorem 5.1 (Weighted dominant energy method). We assume that (HC) holds where $\omega_{c}$ satisfies the PMGC and that $\rho \in C(\bar{\Omega} \times \mathbb{R})$ is nondecreasing with respect to the second variable, and $\rho(\cdot, 0) \equiv 0$ on $\Omega$. Let $\omega_{d}$ be a given subset of $\Omega$ satisfying the PMGC. Let $\phi:[0,+\infty) \mapsto[0,+\infty)$ be a non-increasing and absolutely continuous function. Then, there exist constants $\delta_{i}>0(i=1,2,3)$ and $\alpha^{*}>0$ depending only on $\Omega, \omega_{d}$ but independent of $\phi$ such that for any initial data in the energy space, for all $\alpha^{+} \in\left(0, \alpha^{*}\right]$, the total energy of the system (1.1) satisfies the following nonlinear weighted estimate

$$
\int_{S}^{T} \phi(t) E(t) \mathrm{d} t \leq \delta_{1} \phi(S) E(S)+\delta_{2} \int_{S}^{T} \phi(t) \int_{\Omega} \rho^{2}\left(x, u^{\prime}\right) \mathrm{d} x \mathrm{~d} t+\delta_{3} \int_{S}^{T} \phi(t) \int_{\omega_{d}}\left|u^{\prime}\right|^{2} \mathrm{~d} x \mathrm{~d} t .
$$

Before proving Theorem 5.1, we give a Lemma on a weighted energy estimate for a non-homogeneous wave equation. The Proof of Lemma 5.2 will be given in Section 6 .

Lemma 5.2. Let $\omega$ be a nonempty open subset of $\Omega$ satisfying the PMGC. Let $\phi:[0,+\infty) \mapsto[0,+\infty)$ be a non-increasing and absolutely continuous function. Then, there exist constants $\eta_{i}>0(i=1,2,3,4)$ independent on $\phi$ such that for all $\left(u^{0}, u^{1}\right) \in H_{0}^{1} \times L^{2}$, all $f \in L^{2}\left((0, \infty) ; L^{2}(\Omega)\right)$ and all $0 \leq S \leq T$, the solution $u$ of

$$
\left\{\begin{array}{l}
u^{\prime \prime}-\Delta u=f \quad \text { in } \Omega \times(0,+\infty), \\
u=0 \quad \text { on } \Gamma \times(0,+\infty), \\
\left(u, u^{\prime}\right)(0)=\left(u^{0}, u^{1}\right) \quad \text { in } \Omega
\end{array}\right.
$$


satisfies the estimate

$\int_{S}^{T} \phi(t) e(t) \mathrm{d} t \leq \eta_{1} \phi(S)[e(S)+e(T)]+\eta_{2} \int_{S}^{T}-\phi^{\prime}(t) e(t) \mathrm{d} t+\eta_{3} \int_{S}^{T} \phi(t) \int_{\Omega}|f|^{2} \mathrm{~d} x \mathrm{~d} t+\eta_{4} \int_{S}^{T} \phi(t) \int_{\omega}\left|u^{\prime}\right|^{2} \mathrm{~d} x \mathrm{~d} t$.

where $e(t):=\frac{1}{2} \int_{\Omega}\left(\left|u^{\prime}\right|^{2}+|\nabla u|^{2}\right) \mathrm{d} x$.

Proof of Theorem 5.1. We first consider the case with smooth initial data, then system (1.1) admits a unique solution $(u, v) \in C\left([0,+\infty),\left(H^{2} \cap H_{0}^{1}\right)^{2}\right) \cap W^{1, \infty}\left([0,+\infty),\left(H_{0}^{1}\right)^{2}\right)$.

Let the weight function $\phi$ be a non-increasing absolutely continuous function. Let then

$$
e_{1}(t):=\frac{1}{2} \int_{\Omega}\left(\left|u^{\prime}\right|^{2}+|\nabla u|^{2}\right) \mathrm{d} x
$$

We now apply Lemma 5.2 to the first equation of (1.1) with $\omega=\omega_{d}, f=-\rho\left(., u^{\prime}\right)-\alpha(x) v^{\prime}$, with $E$ given by (1.2) and $e(t)=e_{1}(t)$. Using $e_{1}(t) \leq E(t)$ and the property that $E$ is nonincreasing, we obtain for all $0 \leq S \leq T$ and some constants $\eta_{i}>0(i=1,2,3,4)$ that

$$
\begin{aligned}
\int_{S}^{T} \phi(t) e_{1}(t) \mathrm{d} t \leq & \eta_{1} \phi(S)\left[e_{1}(S)+e_{1}(T)\right]+\eta_{2} \int_{S}^{T}\left(-\phi^{\prime}(t)\right) e_{1}(t) \mathrm{d} t \\
& +2 \eta_{3} \int_{S}^{T} \phi(t) \int_{\Omega} \alpha^{2}(x)\left|v^{\prime}\right|^{2} \mathrm{~d} x \mathrm{~d} t+2 \eta_{3} \int_{S}^{T} \phi(t) \int_{\Omega} \rho^{2}\left(x, u^{\prime}\right) \mathrm{d} x \mathrm{~d} t \\
& +\eta_{4} \int_{S}^{T} \phi(t) \int_{\omega_{d}}\left|u^{\prime}\right|^{2} \mathrm{~d} x \mathrm{~d} t \\
\leq & C_{1} \phi(S) E(S)+2 \eta_{3} \int_{S}^{T} \phi(t) \int_{\Omega} \rho^{2}\left(x, u^{\prime}\right) \mathrm{d} x \mathrm{~d} t \\
& +\eta_{4} \int_{S}^{T} \phi(t) \int_{\omega_{d}}\left|u^{\prime}\right|^{2} \mathrm{~d} x \mathrm{~d} t+2 \eta_{3} \int_{S}^{T} \phi(t) \int_{\Omega} \alpha^{2}(x)\left|v^{\prime}\right|^{2} \mathrm{~d} x \mathrm{~d} t .
\end{aligned}
$$

We now set

$$
e_{2}(t):=\frac{1}{2} \int_{\Omega}\left(\left|v^{\prime}\right|^{2}+|\nabla v|^{2}\right) \mathrm{d} x
$$

and secondly, we apply Lemma 5.2 to the second equation of (1.1) with $\omega=\omega_{c}, f=\alpha(x) u^{\prime}$, with $E$ given by (1.2), $e(t)=e_{2}(t)$. Again, using the inequality $e_{2}(t) \leq E(t)$ and the property that $E$ is nonincreasing, we obtain for all $0 \leq S \leq T$ and some constants $\gamma_{i}>0(i=1,2,3,4)$,

$$
\begin{aligned}
\int_{S}^{T} \phi(t) e_{2}(t) \mathrm{d} t \leq & \gamma_{1} \phi(S)\left[e_{2}(S)+e_{2}(T)\right]+\gamma_{2} \int_{S}^{T}\left(-\phi^{\prime}(t)\right) e_{2}(t) \mathrm{d} t \\
& +\gamma_{3} \int_{S}^{T} \phi(t) \int_{\Omega} \alpha^{2}(x)\left|u^{\prime}\right|^{2} \mathrm{~d} x \mathrm{~d} t+\gamma_{4} \int_{S}^{T} \phi(t) \int_{\omega_{c}}\left|v^{\prime}\right|^{2} \mathrm{~d} t \\
\leq & C_{2} \phi(S) E(S)+\gamma_{4} \int_{S}^{T} \phi(t) \int_{\omega_{c}}\left|v^{\prime}\right|^{2} \mathrm{~d} x \mathrm{~d} t+\gamma_{3} \int_{S}^{T} \phi(t) \int_{\Omega} \alpha^{2}(x)\left|u^{\prime}\right|^{2} \mathrm{~d} x \mathrm{~d} t .
\end{aligned}
$$


Let $\delta>0$ be a real parameter to be chosen later on. Adding (5.6) to $\delta \cdot(5.7)$, we obtain that for all $0 \leq S \leq T$ and all $\delta>0$

$$
\begin{aligned}
\int_{S}^{T} \phi(t)\left(e_{1}(t)+\delta e_{2}(t)\right) \mathrm{d} t \leq & C(1+\delta) \phi(S) E(S)+C \int_{S}^{T} \phi(t) \int_{\Omega} \rho^{2}\left(x, u^{\prime}\right) \mathrm{d} x \mathrm{~d} t \\
& +C \int_{S}^{T} \phi(t) \int_{\omega_{d}}\left|u^{\prime}\right|^{2} \mathrm{~d} x \mathrm{~d} t+C\left(\alpha_{+}+\frac{\delta}{\alpha_{-}}\right) \int_{S}^{T} \phi(t) \int_{\Omega} \alpha\left|v^{\prime}\right|^{2} \mathrm{~d} x \mathrm{~d} t \\
& +C \delta \alpha_{+} \int_{S}^{T} \phi(t) \int_{\Omega} \alpha\left|u^{\prime}\right|^{2} \mathrm{~d} x \mathrm{~d} t,
\end{aligned}
$$

where $C$ denotes generic positive constants which may vary from one line to another. Next, we estimate the term $\int_{S}^{T} \phi(t) \int_{\Omega} \alpha(x)\left|v^{\prime}\right|^{2} \mathrm{~d} x \mathrm{~d} t$ through the coupling relation. Obviously, the following identity holds for the solution $(u, v)$ of system (1.1):

$$
\int_{S}^{T} \phi(t) \int_{\Omega}\left[v^{\prime}\left(u^{\prime \prime}-\Delta u+\alpha(x) v^{\prime}+\rho\left(x, u^{\prime}\right)\right)+u^{\prime}\left(v^{\prime \prime}-\Delta v-\alpha(x) u^{\prime}\right)\right] \mathrm{d} x \mathrm{~d} t=0 .
$$

After integration by parts, we obtain by Cauchy-Schwartz inequality that for all $\varepsilon_{1}>0$

$$
\begin{aligned}
\int_{S}^{T} \phi(t) \int_{\Omega} \alpha(x)\left|v^{\prime}\right|^{2} \mathrm{~d} x \mathrm{~d} t= & \int_{S}^{T} \phi(t) \int_{\Omega} \alpha(x)\left|u^{\prime}\right|^{2} \mathrm{~d} x \mathrm{~d} t-\left[\phi(t) \int_{\Omega}\left(u^{\prime} v^{\prime}+\nabla u \cdot \nabla v\right) \mathrm{d} x\right]_{S}^{T} \\
& +\int_{S}^{T} \phi^{\prime}(t) \int_{\Omega}\left(u^{\prime} v^{\prime}+\nabla u \cdot \nabla v\right) \mathrm{d} x \mathrm{~d} t \\
& -\int_{S}^{T} \phi(t) \int_{\Omega} \rho\left(x, u^{\prime}\right) v^{\prime} \mathrm{d} x \mathrm{~d} t \\
\leq & \int_{S}^{T} \phi(t) \int_{\Omega} \alpha\left|u^{\prime}\right|^{2} \mathrm{~d} x \mathrm{~d} t+C \phi(S) E(S) \\
& +\varepsilon_{1} \int_{S}^{T} \phi(t) \int_{\Omega}\left|v^{\prime}\right|^{2} \mathrm{~d} x \mathrm{~d} t+\frac{C}{\varepsilon_{1}} \int_{S}^{T} \phi(t) \int_{\Omega} \rho^{2}\left(x, u^{\prime}\right) \mathrm{d} x \mathrm{~d} t .
\end{aligned}
$$

Using (5.9) in (5.8), we obtain for all $0<\delta \leq 1$ and all $\varepsilon_{1}>0$ that

$$
\begin{aligned}
\int_{S}^{T} \phi(t)\left(e_{1}(t)+\delta e_{2}(t)\right) \mathrm{d} t \leq & C\left(1+\delta+\alpha_{+}+\frac{\delta}{\alpha_{-}}\right) \phi(S) E(S) \\
& +C\left(1+\left(\alpha_{+}+\frac{\delta}{\alpha_{-}}\right) \frac{1}{\varepsilon_{1}}\right) \int_{S}^{T} \phi(t) \int_{\Omega} \rho^{2}\left(x, u^{\prime}\right) \mathrm{d} x \mathrm{~d} t \\
& +C \int_{S}^{T} \phi(t) \int_{\omega_{d}}\left|u^{\prime}\right|^{2} \mathrm{~d} x \mathrm{~d} t+C_{2}\left(\alpha_{+}+\frac{\delta}{\alpha_{-}}\right) \varepsilon_{1} \int_{S}^{T} \phi(t) \int_{\Omega}\left|v^{\prime}\right|^{2} \mathrm{~d} x \mathrm{~d} t \\
& +C_{1} \alpha_{+}\left(\alpha_{+}+\frac{\delta}{\alpha_{-}}\right) \int_{S}^{T} \phi(t) \int_{\Omega}\left|u^{\prime}\right|^{2} \mathrm{~d} x \mathrm{~d} t,
\end{aligned}
$$

where $C, C_{1}$ and $C_{2}$ are generic positive constants. Thus,

$$
\begin{aligned}
& \left(1-2 C_{1} \alpha_{+}\left(\alpha_{+}+\frac{\delta}{\alpha_{-}}\right)\right) \int_{S}^{T} \phi(t) e_{1}(t) \mathrm{d} t+\left(\delta-2 C_{2} \varepsilon_{1}\left(\alpha_{+}+\frac{\delta}{\alpha_{-}}\right)\right) \int_{S}^{T} \phi(t) e_{2}(t) \mathrm{d} t \\
& \leq C\left(1+\delta+\alpha_{+}+\frac{\delta}{\alpha_{-}}\right) \phi(S) E(S)+C\left(1+\left(\alpha_{+}+\frac{\delta}{\alpha_{-}}\right) \frac{1}{\varepsilon_{1}}\right) \int_{S}^{T} \phi(t) \int_{\Omega} \rho^{2}\left(x, u^{\prime}\right) \mathrm{d} x \mathrm{~d} t \\
& \quad+C \int_{S}^{T} \phi(t) \int_{\omega_{d}}\left|u^{\prime}\right|^{2} \mathrm{~d} x \mathrm{~d} t .
\end{aligned}
$$


Let $\alpha_{+}$be small so that

$$
0<\alpha_{+} \leq \alpha^{*}:=\sqrt{\frac{1}{4 C_{1}}}
$$

We then fix $\delta>0$ so that

$$
\left(1-2 C_{1} \alpha_{+}\left(\alpha_{+}+\frac{\delta}{\alpha_{-}}\right)\right) \geqslant \frac{1}{2}
$$

and choose next $\varepsilon_{1}>0$ so that

$$
\delta-2 C_{2} \varepsilon_{1}\left(\alpha_{+}+\frac{\delta}{\alpha_{-}}\right) \geqslant \frac{\delta}{2}
$$

With these successive choices of $\alpha_{+}, \delta$ and $\varepsilon_{1}$, we deduce that

$$
\begin{aligned}
\int_{S}^{T} \phi(t) E(t) \mathrm{d} t \leq & C\left(1+\delta+\alpha_{+}+\frac{\delta}{\alpha_{-}}\right) \phi(S) E(S) \\
& +C\left(1+\left(\alpha_{+}+\frac{\delta}{\alpha_{-}}\right) \frac{1}{\varepsilon_{1}}\right) \int_{S}^{T} \phi(t) \int_{\Omega} \rho^{2}\left(x, u^{\prime}\right) \mathrm{d} x \mathrm{~d} t \\
& +C \int_{S}^{T} \phi(t) \int_{\omega_{d}}\left|u^{\prime}\right|^{2} \mathrm{~d} x \mathrm{~d} t .
\end{aligned}
$$

For initial data in the energy space, we conclude by a standard argument using density of $\left(H^{2} \cap H_{0}^{1}\right) \times H_{0}^{1}$ in $H_{0}^{1} \times L^{2}$, together with the dissipativity of the underlying nonlinear semigroup. This ends the Proof of Theorem 5.1.

Remark 5.3. The constant $C_{1}$, and therefore the constant $\alpha^{*}$ depends only on $\Omega, \omega_{d}$.

Proof of Theorem 3.2. (Case of polynomially growing feedbacks).

Step 2. Assume that $\left(H F_{p}\right)$ holds.

We first consider the case with smooth initial data, then system (1.1) admits a unique solution $(u, v) \in$ $C\left([0,+\infty),\left(H^{2} \cap H_{0}^{1}\right)^{2}\right) \cap W^{1, \infty}\left([0,+\infty),\left(H_{0}^{1}\right)^{2}\right)$. Moreover, the total energy $E$ defined by $(1.2)$ is absolutely continuous and is non-increasing due to the monotonicity of $\rho$, that is

$$
E^{\prime}(t)=-\int_{\Omega} \rho\left(x, u^{\prime}\right) u^{\prime} \mathrm{d} x \leq 0
$$

Let $t \geqslant 0$ be fixed and $\omega_{d}^{0, t}:=\left\{x \in \omega_{d}:\left|u^{\prime}(t, x)\right| \geqslant 1\right\}$ and $\omega_{d}^{1, t}:=\left\{x \in \omega_{d}:\left|u^{\prime}(t, x)\right| \leq 1\right\}$. In short, we just write $\omega_{d}^{0}, \omega_{d}^{1}$ in the sequel. Then it follows from $\left(\mathrm{HF}_{p}\right)$ and (5.13) that

$$
\int_{S}^{T} \phi(t) \int_{\omega_{d}^{0}}\left|u^{\prime}\right|^{2} \mathrm{~d} x \mathrm{~d} t \leq-C \int_{S}^{T} \phi(t) E^{\prime}(t) \mathrm{d} t \leq C \phi(S) E(S)
$$


Similarly we obtain from $\left(\mathrm{HF}_{p}\right),(5.13)$ and Young inequality that for every $\varepsilon_{2}>0$,

$$
\begin{aligned}
\int_{S}^{T} \phi(t) \int_{\omega_{d}^{1}}\left|u^{\prime}\right|^{2} \mathrm{~d} x \mathrm{~d} t & \leq C \int_{S}^{T} \phi(t) \int_{\Omega}\left|\rho\left(x, u^{\prime}\right) u^{\prime}\right|^{\frac{2}{p+1}} \mathrm{~d} x \mathrm{~d} t \\
& \leq C \int_{S}^{T} \phi(t)\left(\int_{\Omega}\left|\rho\left(x, u^{\prime}\right) u^{\prime}\right| \mathrm{d} x\right)^{\frac{2}{p+1}} \mathrm{~d} t \\
& \leq C \int_{S}^{T} \phi(t)\left(-E^{\prime}(t)\right)^{\frac{2}{p+1}} \mathrm{~d} t \\
& \leq \int_{S}^{T}\left[\varepsilon_{2}(\phi(t))^{\frac{p+1}{p-1}}-C\left(\varepsilon_{2}\right) E^{\prime}(t)\right] \mathrm{d} t \\
& \leq \varepsilon_{2} \int_{S}^{T}(\phi(t))^{\frac{p+1}{p-1}} \mathrm{~d} t+C\left(\varepsilon_{2}\right) E(S),
\end{aligned}
$$

where $C\left(\varepsilon_{2}\right)>0$ stands for a constant depending on $\varepsilon_{2}$ (going to $+\infty$ as $\varepsilon_{2}$ goes to zero). Summing (5.14) and (5.15) gives

$$
\int_{S}^{T} \phi(t) \int_{\omega_{d}}\left|u^{\prime}\right|^{2} \mathrm{~d} x \mathrm{~d} t \leq \max \left(C\left(\varepsilon_{2}\right), C \phi(S)\right) E(S)+\varepsilon_{2} \int_{S}^{T}(\phi(t))^{\frac{p+1}{p-1}} \mathrm{~d} t .
$$

Similarly, let $\Omega^{0}:=\left\{x \in \Omega:\left|u^{\prime}\right| \geqslant 1\right\}$ and $\Omega^{1}:=\left\{x \in \Omega:\left|u^{\prime}\right| \leq 1\right\}$. Note that these subsets depend as above on $t$. We get from $\left(\mathrm{HF}_{p}\right),(5.13)$ and Young inequality that for every $\varepsilon_{3}>0$

$$
\begin{aligned}
\int_{S}^{T} \phi(t) \int_{\Omega} \rho^{2}\left(x, u^{\prime}\right) \mathrm{d} x \mathrm{~d} t & =\int_{S}^{T} \phi(t)\left(\int_{\Omega^{0}} \rho^{2}\left(x, u^{\prime}\right) \mathrm{d} x+\int_{\Omega^{1}} \rho^{2}\left(x, u^{\prime}\right) \mathrm{d} x\right) \mathrm{d} t \\
& \leq C \phi(S) E(S)+\int_{S}^{T} \phi(t) \int_{\Omega}\left|u^{\prime} \rho\left(x, u^{\prime}\right)\right|^{\frac{2}{p+1}} \mathrm{~d} x \mathrm{~d} t \\
& \leq \max \left(C\left(\varepsilon_{3}\right), C \phi(S)\right) E(S)+\varepsilon_{3} \int_{S}^{T}(\phi(t))^{\frac{p+1}{p-1}} \mathrm{~d} t .
\end{aligned}
$$

We now choose the weight function $\phi$ as follows

$$
\phi(t)=E^{\frac{p-1}{2}}(t), \quad \forall t \geqslant 0 .
$$

Combining (5.3), (5.16), (5.17), together with this choice for $\phi$, and letting $\varepsilon_{2}, \varepsilon_{3}$ small enough, we obtain that for all $0 \leq S \leq T$,

$$
\int_{S}^{T} E^{\frac{p+1}{2}}(t) \mathrm{d} t \leq \max \left(C_{1}, C_{2} E^{\frac{p-1}{2}}(0)\right) E(S),
$$

where $C_{1}, C_{2}$ are positive constants independent of $E(0)$.

We finish the proof by Step 3, which says that nonnegative, nonincreasing functions $E$ satisfying a polynomial nonlinear integral inequality, then satisfy a polynomial decay rate. Applying Corollary 4.5 with $\alpha=\frac{p-1}{2}>0$, and $M=C\left(1+E^{\frac{p-1}{2}}(0)\right)$, we end the Proof of Theorem 3.2 and obtain when $E(0)>0$

$$
E(t) \leq C_{E(0)} t^{-2 /(p-1)}, \quad \forall t \geqslant C_{1} E^{\frac{-(p-1)}{2}}(0)+C_{2},
$$

where

$$
C_{E(0)}=\left(\max \left(C_{1}, C_{2} E^{\frac{p-1}{2}}(0)\right)\left(1+\frac{1}{\alpha}\right)\right)^{1 / \alpha}
$$


Proof of Theorem 3.4. (Case of general growing feedbacks).

In Step 2, We shall prove the following theorem

Theorem 5.4 (Optimal-weight convexity method). Assume the hypotheses of Theorem 5.1. Assume furthermore that $\left(H F_{g}\right)$ holds where $g$ is such that the function $H$ defined in $(2.3)$ is strictly convex on $\left[0, r_{0}^{2}\right]$, and $g^{\prime}(0)=0$. We define $L$ by (2.4). Let the initial data be in the energy space and be non vanishing, $(u, v)$ be the solution of (1.1) and $E$ be its energy. Then $E$ satisfies the following nonlinear weighted integral inequality

$$
\int_{S}^{T} L^{-1}\left(\frac{E(t)}{2 \beta}\right) E(t) \mathrm{d} t \leq M E(S), \quad \forall 0 \leq S \leq T,
$$

where $\beta$ and $M$ are respectively given by:

$$
\beta=\max \left(C_{2}, \frac{E(0)}{2 L\left(H^{\prime}\left(r_{0}^{2}\right)\right)}\right),
$$

and

$$
M=2 C_{1}\left(1+H^{\prime}\left(r_{0}^{2}\right)\right),
$$

with $C_{1}>0, C_{2}>0$ depending on $\delta_{i}(i=1,2,3), \Omega, \omega_{d}$ but independent of $E(0)$.

Remark 5.5. This method is called the optimal-weight convexity method according to the property that the weight function $\phi$ is chosen in an optimal way by setting

$$
\phi(.)=L^{-1}\left(\frac{E(.)}{2 \beta}\right)
$$

thanks to suitable convexity arguments relying both on Jensen and Young's inequalities for an appropriate convex function.

Proof. We consider as before smooth initial data, then the solution $(u, v)$ of $(1.1)$ is in $C\left([0,+\infty),\left(H^{2} \cap\right.\right.$ $\left.\left.H_{0}^{1}\right)^{2}\right) \cap W^{1, \infty}\left([0,+\infty),\left(H_{0}^{1}\right)^{2}\right)$. Moreover, the total energy $E$ satisfies the dissipation relation (5.13). Thanks to Theorem 5.1, we know that $E$ satisfies the weighted dominant energy estimate (5.3). We shall now use the optimal-weight convexity method of the first author [4] to build an optimal weight function $\phi$ to prove that the two terms

$$
\int_{S}^{T} \phi(t) \int_{\Omega} \rho^{2}\left(x, u^{\prime}\right) \mathrm{d} x \mathrm{~d} t \text { and } \int_{S}^{T} \phi(t) \int_{\omega_{d}}\left|u^{\prime}\right|^{2} \mathrm{~d} x \mathrm{~d} t
$$

in (5.3), are bounded above by the term

$$
C E(S)(1+\phi(S))+C \int_{S}^{T} E(t) \phi(t) \mathrm{d} t \quad \forall 0 \leq S \leq T .
$$

We proceed as in [4]. Choose a parameter $\varepsilon_{0}$ sufficiently small, e.g. $\varepsilon_{0}=\min \left(1, g\left(r_{0}\right)\right)$.

For fixed $t \geqslant 0$, we define the subset $\Omega_{1}^{t}=\left\{x \in \Omega,\left|u^{\prime}(t, x)\right| \leq \varepsilon_{0}\right\}$. Now thanks to (HF ${ }_{g}$ ), we know that $(2.2)$ holds. Hence, since $g$ is increasing, we have

$$
g\left(\frac{\left|\rho\left(x, u^{\prime}(t, x)\right)\right|}{K}\right) \leq\left|u^{\prime}(t, x)\right|, \text { for a.e } x \in \Omega_{1}^{t},
$$

where $K=c_{2}\|a\|_{\infty}$, with $\|\cdot\|_{\infty}$ standing for the $L^{\infty}$ norm. Now, we can note that parameter $\varepsilon_{0}$ has been chosen to guarantee the following two properties

$$
\frac{1}{\left|\Omega_{1}^{t}\right|} \int_{\Omega_{1}^{t}} \frac{\left|\rho\left(x, u^{\prime}(t, x)\right)\right|^{2}}{K^{2}} \mathrm{~d} x \in\left[0, r_{0}^{2}\right],
$$


and

$$
\frac{1}{\left|\Omega_{1}^{t}\right| K} \int_{\Omega_{1}^{t}} \rho\left(x, u^{\prime}(t, x)\right) u^{\prime}(t, x) \mathrm{d} x \in\left[0, H\left(r_{0}^{2}\right)\right],
$$

hold. Since $H$ has been assumed to be convex on $\left[0, r_{0}^{2}\right]$ and thanks to (5.22), the Jensen's inequality, and (5.21), we obtain

$$
\begin{aligned}
H\left(\frac{1}{\left|\Omega_{1}^{t}\right|} \int_{\Omega_{1}^{t}} \frac{\left|\rho\left(x, u^{\prime}(t, x)\right)\right|^{2}}{K^{2}} \mathrm{~d} x\right) & \leq \frac{1}{\left|\Omega_{1}^{t}\right|} \int_{\Omega_{1}^{t}} H\left(\frac{\left|\rho\left(x, u^{\prime}(t, x)\right)\right|^{2}}{K^{2}}\right) \mathrm{d} x \\
& =\frac{1}{\left|\Omega_{1}^{t}\right| K} \int_{\Omega_{1}^{t}}\left|\rho\left(u^{\prime}(t, x)\right)\right| g\left(\frac{\left|\rho\left(x, u^{\prime}(t, x)\right)\right|}{K}\right) \mathrm{d} x \\
& \leq \frac{1}{\left|\Omega_{1}^{t}\right| K} \int_{\Omega_{1}^{t}} u^{\prime}(t, x) \rho\left(x, u^{\prime}(t, x)\right) \mathrm{d} x .
\end{aligned}
$$

By (5.23), we deduce that

$$
\int_{S}^{T} \phi(t) \int_{\Omega_{1}^{t}}\left|\rho\left(x, u^{\prime}(t, x)\right)\right|^{2} \mathrm{~d} x \mathrm{~d} t \leq \int_{S}^{T} K^{2}\left|\Omega_{1}^{t}\right| \phi(t) H^{-1}\left(\frac{1}{\left|\Omega_{1}^{t}\right| K} \int_{\Omega_{1}^{t}} u^{\prime}(t, x) \rho\left(x, u^{\prime}(t, x)\right) \mathrm{d} x\right) \mathrm{d} t,
$$

and using further Young's inequality, the dissipation relation (5.13), we obtain

$$
\int_{S}^{T} \phi(t) \int_{\Omega_{1}^{t}}\left|\rho\left(x, u^{\prime}(t, x)\right)\right|^{2} \mathrm{~d} x \mathrm{~d} t \leq K^{2}|\Omega| \int_{S}^{T} \widehat{H}^{*}(\phi(t)) \mathrm{d} t+K E(S), \quad \forall 0 \leq S \leq T .
$$

On the other hand, we prove easily as in the Proof of Theorem 3.2 that

$$
\int_{S}^{T} \phi(t) \int_{\Omega \backslash \Omega_{1}^{t}}\left|\rho\left(x, u^{\prime}(t, x)\right)\right|^{2} \mathrm{~d} x \mathrm{~d} t \leq K E(S) \phi(S), \quad \forall 0 \leq S \leq T .
$$

Adding these two inequalities, we obtain

$$
\int_{S}^{T} \phi(t) \int_{\Omega}\left|\rho\left(x, u^{\prime}(t, x)\right)\right|^{2} \mathrm{~d} x \mathrm{~d} t \leq K^{2}|\Omega| \int_{S}^{T} \widehat{H}^{*}(\phi(t)) \mathrm{d} t+K E(S)(1+\phi(S)), \quad \forall 0 \leq S \leq T .
$$

We now turn to the estimate of the localized weighted linear kinetic energy. Thanks to $\left(\mathrm{HF}_{g}\right)$, we know that $(2.2)$ holds. Choose a parameter $\varepsilon_{1}$ sufficiently small, e.g. $\varepsilon_{1}=\min \left\{r_{0}, g\left(r_{1}\right)\right\}$ where $r_{1}$ is defined by

$$
r_{1}^{2}=H^{-1}\left(\frac{k}{K} H\left(r_{0}^{2}\right)\right)
$$

where $k=c_{1} a_{-}$. For fixed $t \geqslant 0$, we define the subset $\omega_{d}^{t}=\left\{x \in \omega_{d},\left|u^{\prime}(t, x)\right| \leq \varepsilon_{1}\right\}$. Thanks to (2.2) and since $\left(\mathrm{HF}_{g}\right)$ holds, we have

$$
g\left(\left|u^{\prime}(t, x)\right|\right) \leq \frac{\left|\rho\left(x, u^{\prime}(t, x)\right)\right|}{k}, \text { for a.e } x \in \omega_{d}^{t} .
$$

Now, we can note that parameter $\varepsilon_{1}$ has been chosen to guarantee the following two properties

$$
\frac{1}{\left|\omega_{d}^{t}\right|} \int_{\omega_{d}^{t}}\left|u^{\prime}(t, x)\right|^{2} \mathrm{~d} x \in\left[0, r_{0}^{2}\right]
$$

and

$$
\frac{1}{\left|\omega_{d}^{t}\right| k} \int_{\omega_{d}^{t}} \rho\left(x, u^{\prime}(t, x)\right) u^{\prime}(t, x) \mathrm{d} x \in\left[0, H\left(r_{0}^{2}\right)\right]
$$


hold. Since $H$ has been assumed to be convex on $\left[0, r_{0}^{2}\right]$ and thanks to (5.26), the Jensen's inequality, and (5.25), we obtain

$$
\begin{aligned}
H\left(\frac{1}{\left|\omega_{d}^{t}\right|} \int_{\omega_{d}^{t}}\left|u^{\prime}(t, x)\right|^{2} \mathrm{~d} x\right) & \leq \frac{1}{\left|\omega_{d}^{t}\right|} \int_{\omega_{d}^{t}} H\left(\left|u^{\prime}(t, x)\right|^{2}\right) \mathrm{d} x \\
& =\frac{1}{\left|\omega_{d}^{t}\right|} \int_{\omega_{d}^{t}}\left|u^{\prime}(t, x)\right| g\left(\left|u^{\prime}(t, x)\right|\right) \mathrm{d} x \\
& \leq \frac{1}{\left|\omega_{d}^{t}\right| k} \int_{\omega_{d}^{t}} u^{\prime}(t, x) \rho\left(x, u^{\prime}(t, x)\right) \mathrm{d} x
\end{aligned}
$$

Thanks to (5.27), we deduce that

$$
\int_{S}^{T} \phi(t) \int_{\omega_{d}^{t}}\left|u^{\prime}(t, x)\right|^{2} \mathrm{~d} x \mathrm{~d} t \leq \int_{S}^{T}\left|\omega_{d}^{t}\right| \phi(t) H^{-1}\left(\frac{1}{\left|\omega_{d}^{t}\right| k} \int_{\Omega_{1}^{t}} u^{\prime}(t, x) \rho\left(x, u^{\prime}(t, x)\right) \mathrm{d} x\right) \mathrm{d} t,
$$

and using further Young's inequality, the dissipation relation (5.13), we obtain

$$
\int_{S}^{T} \phi(t) \int_{\omega_{d}^{t}}\left|u^{\prime}(t, x)\right|^{2} \mathrm{~d} x \mathrm{~d} t \leq\left|\omega_{d}\right| \int_{S}^{T} \widehat{H}^{*}(\phi(t)) \mathrm{d} t+\frac{1}{k} E(S), \quad \forall 0 \leq S \leq T .
$$

On the other hand, we prove easily as in the Proof of Theorem 3.2 that

$$
\int_{S}^{T} \phi(t) \int_{\omega_{d} \backslash \omega_{d}^{t}}\left|u^{\prime}(t, x)\right|^{2} \mathrm{~d} x \mathrm{~d} t \leq \frac{1}{k} E(S) \phi(S), \quad \forall 0 \leq S \leq T .
$$

Adding these two inequalities, we obtain

$$
\int_{S}^{T} \phi(t) \int_{\omega_{d}}\left|u^{\prime}(t, x)\right|^{2} \mathrm{~d} x \mathrm{~d} t \leq\left|\omega_{d}\right| \int_{S}^{T} \widehat{H}^{*}(\phi(t)) \mathrm{d} t+\frac{1}{k} E(S)(1+\phi(S)), \quad \forall 0 \leq S \leq T .
$$

Using (5.24) and (5.28) in the weighted dominant energy estimate (5.3), we obtain

$$
\int_{S}^{T} \phi(t) E(t) \mathrm{d} t \leq C_{1} E(S)(1+\phi(S))+C_{2} \int_{S}^{T} \widehat{H}^{*}(\phi(t)) \mathrm{d} t, \quad \forall 0 \leq S \leq T,
$$

where the constants $C_{1}, C_{2}$ depend only on the $\delta_{i}$ for $i=1,2,3$ and on $|\Omega|$ and $\left|\omega_{d}\right|$ in an explicit way. In particular, they do not depend on $\phi$.

Let

$$
\beta=\max \left(C_{2}, \frac{E(0)}{2 L\left(H^{\prime}\left(r_{0}^{2}\right)\right)}\right),
$$

where $L$ is defined in (2.4). Since $E$ is a nonincreasing function, and thanks to (2.5), we have

$$
\frac{E(t)}{2 \beta} \leq \frac{E(0)}{2 \beta} \leq L\left(H^{\prime}\left(r_{0}^{2}\right)\right)<r_{0}^{2}
$$

Hence, since $L^{-1}$ is defined from $\left[0, r_{0}^{2}\right)$ onto $[0,+\infty)$, we can define $\phi$ by

$$
\phi(t)=L^{-1}\left(\frac{E(t)}{2 \beta}\right), \quad \forall t \geqslant 0
$$


By definition of $L, \phi$ is a nonnegative, non increasing and absolutely continuous function on $[0,+\infty)$. We first note that

$$
\phi(S) \leq H^{\prime}\left(r_{0}^{2}\right), \quad \forall S \geqslant 0 .
$$

Then, thanks to our "optimal" choice of the weight function $\phi$ and to the definition of $L$, we have

$$
L(\phi(t))=\frac{E(t)}{2 \beta}=\frac{\widehat{H}^{*}(\phi(t))}{\phi(t)}, \quad \forall t \geqslant 0 .
$$

This implies

$$
C_{2} \widehat{H}^{*}(\phi(t)) \leq \beta \widehat{H}^{*}(\phi(t))=\frac{1}{2} \phi(t) E(t), \quad \forall t \geqslant 0 .
$$

Combining this estimate together with (5.32) in (5.29), we obtain

$$
\int_{S}^{T} L^{-1}\left(\frac{E(t)}{2 \beta}\right) E(t) \mathrm{d} t \leq M E(S), \quad \forall 0 \leq S \leq T,
$$

where

$$
M=2 C_{1}\left(1+H^{\prime}\left(r_{0}^{2}\right)\right) .
$$

We finish the Proof of Theorem 3.4 by Step 3. Thanks to Step 2, $E$ satisfies the weighted nonlinear integral inequality (5.33), where $\beta$ is defined by (5.30), $M$ is defined by (5.34). Hence applying Theorem 4.8 with this $\beta$ and $M$ we deduce that $E$ satisfies the decay rate (4.7) in the general case, and the simplified decay rate (4.8) if $\limsup \Lambda_{H}(x)<1$. This concludes the Proof of Theorem 3.4.

Remark 5.6. The smallness of $\alpha$ can be reduced if we assume additionally $\operatorname{supp}\{\alpha\} \subset \omega_{d}$ in Theorem 3.4. Actually, we can choose $\delta=1$ in the proof of (5.10), and in that case, the last term of (5.10) can be replaced by

$$
C_{1} \int_{S}^{T} \phi(t) \int_{\omega_{d}}\left|u^{\prime}\right|^{2} \mathrm{~d} x \mathrm{~d} t
$$

Then (5.3) follows easily by choosing $\varepsilon_{1}>0$ sufficiently small in (5.10). The proof afterwards is the same.

\subsection{Proof of Theorem 3.8}

Thanks to our hypotheses, the simplified upper energy estimate (4.8) of Theorem 4.8 holds, so that $E(t)$ converges to 0 as $t$ goes to infinity. Hence, there exists $T_{0} \geqslant 0$ such that

$$
E(t) \leq\left(\frac{r_{0}^{2}}{\gamma_{s}}\right)^{2}, \quad \forall t \geq T_{0},
$$

where $\gamma_{s}=4 \sqrt{E_{1}(0)}$. Hence

$$
\gamma_{s} \sqrt{E(t)} \in\left[0, r_{0}^{2}\right] \text { for all } t \geqslant T_{0} .
$$

On the other hand, thanks to the regularity of $u$ (see [9] for details), we have

$$
\left\|u^{\prime}(t, .)\right\|_{L^{\infty}(\Omega)} \leq \gamma_{s} \sqrt{E(t)} .
$$

Using this inequality in the dissipation relation

$$
-E^{\prime}(t)=\int_{\Omega} a(x) u^{\prime} g\left(u^{\prime}\right) \mathrm{d} x, \quad t \geqslant 0,
$$


together with (5.36), we deduce that for all $t \geqslant T_{0}$

$$
-E^{\prime}(t) \leq\|a\|_{L^{\infty}(\Omega)} \frac{2}{\gamma_{s}} \sqrt{E(t)} H\left(\gamma_{s} \sqrt{E(t)}\right) .
$$

Therefore, we have

$$
E(t) \geq\left(\frac{1}{\gamma_{s}} K^{-1}\left(\|a\|_{L^{\infty}(\Omega)}\left(t-T_{0}\right)\right)\right)^{2}, \quad \forall t \geqslant T_{0},
$$

where $K^{-1}$ denotes the inverse function of $K$ defined by

$$
K(\tau)=\int_{\tau}^{z_{0}} \frac{d y}{H(y)}, \quad \forall \tau \in\left(0, \sqrt{E\left(T_{0}\right)}\right)
$$

where

$$
z_{0}=\gamma_{s} \sqrt{E\left(T_{0}\right)}
$$

We denote by $z$ the solution of the following ordinary differential equation

$$
z^{\prime}(t)+\|a\|_{L^{\infty}(\Omega)} H(z(t))=0, z(0)=z_{0} .
$$

Then we have the relation

$$
z\left(t-T_{0}\right)=K^{-1}\left(\|a\|_{L^{\infty}(\Omega)}\left(t-T_{0}\right)\right), \quad \forall t \geqslant T_{0} .
$$

We now use the following comparison Lemma, that we recall for the sake of completeness.

Lemma 5.7 ([7], Lem. 2.4). Let $H$ be a given strictly convex $\mathcal{C}^{1}$ function from $\left[0, r_{0}^{2}\right]$ to $\mathbb{R}$ such that $H(0)=$ $H^{\prime}(0)=0$, where $r_{0}>0$ is sufficiently small and define $\Lambda_{H}$ on $\left(0, r_{0}^{2}\right]$ by $(2.6)$.

Let $z$ be the solution of the ordinary differential equation:

$$
z^{\prime}(t)+\kappa H(z(t))=0, z(0)=z_{0} \quad t \geq 0,
$$

where $z_{0}>0$ and $\kappa>0$ are given. Then $z(t)$ is defined for every $t \geq 0$ and decays to 0 at infinity. Moreover assume that $\left(H F_{l}\right)$ holds. Then there exists $T_{1}>0$ such that for all $R>0$ there exists a constant $C>0$ such that

$$
z(t) \geq C\left(H^{\prime}\right)^{-1}\left(\frac{R}{t}\right), \quad \forall t \geq T_{1}
$$

where $T_{1}$ is a positive constant.

We apply this Lemma to the solution $z$ of (5.40) with $R=1$ and $\kappa=\|a\|_{L^{\infty}(\Omega)}$. Thus, there exist two constants $T_{1}>0$ and $C_{s}>0$ such that

$$
z(t) \geq C_{s}\left(H^{\prime}\right)^{-1}\left(\frac{1}{t}\right), \quad \forall t \geq T_{1}
$$

Combining (5.44) together with (5.37) and (5.41), we obtain the lower estimate (3.8).

Remark 5.8. The constant $C$ of the above Lemma 5.7 depends explicitly on $\kappa, R$ (and in addition of $\mu$ if the second alternative of $\left(H F_{l}\right)$ holds). This dependence is given in the proof of Lemma 2.4 in [7]. Moreover, Lemma 5.7 is also true for $r_{0}=\infty$. In this case the interval $\left[0, r_{0}^{2}\right]$ becomes $[0,+\infty)$. 


\subsection{Proof of the decay rates given in Examples 3.11-3.14}

Example 3.11. We have $H(x)=x^{(p+1) / 2}$ for $x \in\left[0, r_{0}^{2}\right]$. Thus $H^{\prime}(x)=\frac{p+1}{2} x^{(p-1) / 2}$ and $H$ is strictly convex on a right neighborhood of 0 . Moreover, $\Lambda_{H}(x)=\frac{2}{p+1}<1$ for all $x \in\left[0, r_{0}^{2}\right]$. We easily conclude applying (4.8) of Theorems 4.8 and 3.8 for the lower estimate in the one-dimensional case.

Example 3.12. We have $H(x)=\sqrt{x} \mathrm{e}^{\frac{-1}{x}}$ for $x \in\left[0, r_{0}^{2}\right]$. Thus $H^{\prime}(x)=\frac{\mathrm{e}^{-1 / x}}{\sqrt{x}}\left(\frac{1}{2}+\frac{1}{x}\right)$, and $H$ is strictly convex on a right neighborhood of 0 . Moreover, we have $\Lambda_{H}(x)=\frac{1}{\left(\frac{1}{2}+\frac{1}{x}\right)}$ for all $x>0$ sufficiently close to 0 , so that $\lim _{x \rightarrow 0+} \Lambda_{H}(x)=0$. We apply (4.8) of Theorem 4.8. So we set $x(t)=\left(H^{\prime}\right)^{-1}\left(\frac{\kappa M}{t}\right)$. Then one can prove that $x(t)$ is equivalent to $\frac{1}{\ln (t)-\ln (\kappa M)}$ as $t$ goes to $+\infty$. We therefore obtain the desired upper bound, using this equivalence. One can show that the second alternative of $\left(H F_{l}\right)$ holds for any $\mu>1$ (see Sect. 7.10 in [7]). Thus, we obtain in the same way by Theorem 3.8 the lower estimates in the one-dimensional case.

Example 3.13. We have $H(x)=x^{(p+1) / 2}(\ln (1 / \sqrt{x}))^{q}$ for $x \in\left[0, r_{0}^{2}\right]$. Thus $H$ is strictly convex on a right neighborhood of 0 and $H^{\prime}(x)=\frac{1}{2} x^{(p-1) / 2}(\ln (1 / \sqrt{x}))^{q}\left(p+1-q(\ln (1 / \sqrt{x}))^{-1}\right)$. Moreover, $\Lambda_{H}(x)=\frac{2}{p+1-q(\ln (1 / \sqrt{x}))^{-1}}$ for all $x>0$ sufficiently close to 0 , so that $\lim _{x \rightarrow 0+} \Lambda_{H}(x)=\frac{2}{p+1}$. We apply (4.8) of Theorem 4.8. So we set $x(t)=\left(H^{\prime}\right)^{-1}\left(\frac{\kappa M}{t}\right)$ and $y(t)=\left(\frac{2 \kappa M}{t}\right)^{2 /(p-1)}$. Then one can prove that $\left(\frac{x(t)}{y(t)}\right)^{(p-1) / 2}(\ln (1 / \sqrt{x}))^{q}$ is equivalent to $\frac{1}{p+1}$ as $t$ goes to $+\infty$. On the other hand, computing $\ln (x(t))$ and $\ln (y(t))$, we find that $\ln (x(t))$ is equivalent to $\ln (y(t))$ as $t$ goes to $+\infty$. Using this relation in the previous one, we find that $x(t)$ is equivalent to $D t^{-2 /(p-1)}(\ln (t))^{-2 q /(p-1)}$, where $D$ is an explicit positive constant which depends on $\kappa, M, p$ and $q$. We therefore obtain the desired upper estimate. The lower estimates in the one-dimensional case follows from Theorem 3.8.

Example 3.14. We have $H(x)=\sqrt{x} \mathrm{e}^{-(\ln (1 / \sqrt{x}))^{p}}$ for $x \in\left[0, r_{0}^{2}\right]$. Thus, $H$ is strictly convex on a right neighborhood of 0 and $H^{\prime}(x)=\frac{1}{2 \sqrt{x}} \mathrm{e}^{-(\ln (1 / \sqrt{x}))^{p}}\left(1+p\left(\ln \left(\frac{1}{\sqrt{x}}\right)\right)^{p-1}\right)$. Moreover, we have $\Lambda_{H}(x)=\frac{2}{1+p(\ln (1 / \sqrt{x}))^{p-1}}$ for all $x>0$ sufficiently close to 0 , so that $\lim _{x \rightarrow 0+} \Lambda_{H}(x)=0$. We apply (4.8) of Theorem 4.8. So we set $x(t)=\left(H^{\prime}\right)^{-1}\left(\frac{\kappa M}{t}\right)$ and $y(t)=\mathrm{e}^{-2\left(\ln \left(\frac{t}{\kappa M}\right)\right)^{1 / p}}$. Then one can prove that $\ln (x(t))$ is equivalent to $\ln (y(t))$ as $t$ goes to $+\infty$. We further set $z(t)=\ln (1 / \sqrt{x(t)})$ so that $z(t)$ goes to $+\infty$ as $t$ goes to $+\infty$, then we have $z^{p}(t)(1-\theta(t))=\ln \left(\frac{t}{2 \kappa M}\right)$, where $\theta(t)=z^{1-p}(t)+\ln \left(1+p z^{p-1}(t)\right) z^{-p}(t)$, so that $\theta(t)$ goes to 0 as $t$ goes to $+\infty$. Hence we have $x(t)=\mathrm{e}^{-2\left(\ln \left(\frac{t}{\kappa M}\right)\right)^{1 / p} \frac{1}{(1-\theta(t))^{1 / p}}}$. We can check that $\ln \left(\frac{t}{2 \kappa M}\right)^{1 / p}\left(1-(1-\theta(t))^{1 / p}\right)$ goes to 0 as $t$ goes to $+\infty$. Hence, $x(t)$ is equivalent to $\mathrm{e}^{-2(\ln (t))^{1 / p}}$ as $t$ goes to $+\infty$. We therefore obtain the desired upper estimate. One can show that the second alternative of $\left(H F_{l}\right)$ holds for any $\mu>1$ (see Sect. 7.10 in [7]). Thus, we obtain by Theorem 3.8 the lower estimates in the one-dimensional case.

\section{Proof of Lemma 5.2}

In this section, we give a Proof of Lemma 5.2 based on the piecewise multiplier method which relies on the geometric assumptions $P M G C$ on the subset $\omega \subset \Omega$ (introduced for the first time in [31], see also [33,34]). Our Lemma concerns a forced wave equation, and is not formulated under this form in the literature. For the sake of completeness, we give its proof below. Denoting by $\Omega_{j}$ and $x_{j}(j=1, \ldots, J)$ the sets and the points given by the $P M G C$, we have $\omega \supset N_{\varepsilon}\left(\cup_{j=1}^{J} \gamma_{j}\left(x_{j}\right) \cup\left(\Omega \backslash \cup_{j=1}^{J} \Omega_{j}\right)\right) \cap \Omega$. Here, $N_{\varepsilon}(U)=\left\{x \in \mathbb{R}^{n}, d(x, U) \leq \varepsilon\right\}$ with $d(\cdot, U)$ the usual euclidian distance to the subset $U$ of $\mathbb{R}^{n}$, and $\gamma_{j}\left(x_{j}\right)=\left\{x \in \Gamma_{j},\left(x-x_{j}\right) \cdot \nu_{j}>0\right\}$, where $\nu_{j}$ denotes the outward unit normal of the boundary $\Gamma_{j}=\partial \Omega_{j}$.

Let $0<\varepsilon_{0}<\varepsilon_{1}<\varepsilon_{2}<\varepsilon$ and define $Q_{i}:=N_{\varepsilon_{i}}\left[\cup_{j=1}^{J} \gamma_{j}\left(x_{j}\right) \cup\left(\Omega \backslash \cup_{j=1}^{J} \Omega_{j}\right)\right](i=0,1,2)$. Since $\left(\overline{\Omega_{j}} \backslash Q_{1}\right) \cap \overline{Q_{0}}=\emptyset$, we introduce a cut-off function $\psi_{j} \in C_{0}^{\infty}\left(\mathbb{R}^{N}\right)$ satisfying

$$
0 \leq \psi_{j} \leq 1, \quad \psi_{j}=1 \quad \text { on } \overline{\Omega_{j}} \backslash Q_{1} ; \quad \psi_{j}=0 \quad \text { on } Q_{0} .
$$


For $m_{j}(x)=x-x_{j}$, we define the $C^{1}$ vector field on $\Omega$ :

$$
h(x)=\left\{\begin{array}{l}
\psi_{j}(x) m_{j}(x) \quad \text { if } x \in \Omega_{j}, j=1, \ldots, J \\
0 \quad \text { if } x \in \Omega \backslash \cup_{j=1}^{J} \Omega_{j} .
\end{array}\right.
$$

Using the multiplier $\phi(t) h(x) \cdot \nabla u$ to equation (5.4):

$$
\int_{S}^{T} \phi(t) \int_{\Omega_{j}} h(x) \cdot \nabla u\left(u^{\prime \prime}-\Delta u-f\right) \mathrm{d} x \mathrm{~d} t=0
$$

leads to

$$
\begin{aligned}
\int_{S}^{T} \phi(t) \int_{\Gamma_{j}} \frac{\partial u}{\partial \nu_{j}} h \cdot \nabla u+\frac{1}{2}\left(h \cdot \nu_{j}\right)\left(\left|u^{\prime}\right|^{2}-|\nabla u|^{2}\right) d \Gamma \mathrm{d} t= & {\left[\phi(t) \int_{\Omega_{j}} u^{\prime} h \cdot \nabla u \mathrm{~d} x\right]_{S}^{T}-\int_{S}^{T} \phi^{\prime}(t) \int_{\Omega_{j}} u^{\prime} h \cdot \nabla u \mathrm{~d} x \mathrm{~d} t } \\
& +\int_{S}^{T} \phi(t) \int_{\Omega_{j}} \frac{1}{2} \operatorname{divh}\left(\left|u^{\prime}\right|^{2}-|\nabla u|^{2}\right) \mathrm{d} x \mathrm{~d} t \\
& +\int_{S}^{T} \phi(t) \int_{\Omega_{j}}\left[\sum_{i, k} \frac{\partial u}{\partial x_{i}} \frac{\partial u}{\partial x_{k}} \frac{\partial h_{k}}{\partial x_{i}}-h \cdot \nabla u f\right] \mathrm{d} x \mathrm{~d} t .
\end{aligned}
$$

Thanks to the choice of $\psi_{j}$, the terms in the left hand side of (6.3) vanish except on the boundary $\left(\Gamma_{j} \backslash \gamma_{j}\left(x_{j}\right)\right) \cap \Gamma$. Since $u=0$ on this part of boundary, then $u^{\prime}=0, \nabla u=\frac{\partial u}{\partial \nu_{j}} \nu_{j}$. Hence, the left side of (6.3) becomes

$$
\frac{1}{2} \int_{S}^{T} \phi(t) \int_{\left(\Gamma_{j} \backslash \gamma_{j}\left(x_{j}\right)\right) \cap \Gamma}\left|\frac{\partial u}{\partial \nu_{j}}\right|^{2} \psi_{j} m_{j} \cdot \nu_{j} d \Gamma \mathrm{d} t \leq 0 .
$$

Therefore (6.3) implies that

$$
\begin{aligned}
\int_{S}^{T} \phi(t) & \int_{\Omega_{j}} \frac{1}{2} \operatorname{div} h\left(\left|u^{\prime}\right|^{2}-|\nabla u|^{2}\right) \mathrm{d} x \mathrm{~d} t+\int_{S}^{T} \phi(t) \int_{\Omega_{j}} \sum_{i, k} \frac{\partial u}{\partial x_{i}} \frac{\partial u}{\partial x_{k}} \frac{\partial h_{k}}{\partial x_{i}} \mathrm{~d} x \mathrm{~d} t \\
\leq & -\left[\phi(t) \int_{\Omega_{j}} u^{\prime} h \cdot \nabla u \mathrm{~d} x\right]_{S}^{T}+\int_{S}^{T} \phi^{\prime}(t) \int_{\Omega_{j}} u^{\prime} h \cdot \nabla u \mathrm{~d} x \mathrm{~d} t \\
& +\int_{S}^{T} \phi(t) \int_{\Omega_{j}} h \cdot \nabla u f \mathrm{~d} x \mathrm{~d} t .
\end{aligned}
$$

Since, moreover $h(x)=m_{j}(x)$ on $\bar{\Omega}_{j} \backslash Q_{1}$, we obtain that

$$
\begin{aligned}
\int_{S}^{T} \phi(t) \int_{\Omega_{j} \backslash Q_{1}} \frac{N}{2}\left(\left|u^{\prime}\right|^{2}-|\nabla u|^{2}\right)+|\nabla u|^{2} \mathrm{~d} x \mathrm{~d} t \leq & -\left[\phi(t) \int_{\Omega_{j}} u^{\prime} h \cdot \nabla u \mathrm{~d} x\right]_{S}^{T}+\int_{S}^{T} \phi^{\prime}(t) \int_{\Omega_{j}} u^{\prime} h \cdot \nabla u \mathrm{~d} x \mathrm{~d} t \\
& +\int_{S}^{T} \phi(t) \int_{\Omega_{j}} h \cdot \nabla u f \mathrm{~d} x \mathrm{~d} t \\
& -\int_{S}^{T} \phi \int_{\Omega_{j} \cap Q_{1}}\left[\frac{1}{2} \operatorname{divh}\left(\left|u^{\prime}\right|^{2}-|\nabla u|^{2}\right)+\sum_{i, k} \frac{\partial u}{\partial x_{i}} \frac{\partial u}{\partial x_{k}} \frac{\partial h_{k}}{\partial x_{i}}\right] \mathrm{d} x \mathrm{~d} t .
\end{aligned}
$$


Summing the above inequality on $j$ and using the facts that $\Omega \backslash Q_{1}=\cup_{j=1}^{J}\left(\Omega_{j}\right) \backslash Q_{1}$ and $h(x)=0$ on $\Omega \backslash \cup_{j=1}^{J} \Omega_{j}$, we obtain

$$
\begin{aligned}
\int_{S}^{T} \phi(t) \int_{\Omega \backslash Q_{1}} \frac{N}{2}\left(\left|u^{\prime}\right|^{2}-|\nabla u|^{2}\right)+|\nabla u|^{2} \mathrm{~d} x \mathrm{~d} t \leq & -\left[\phi(t) \int_{\Omega} u^{\prime} h \cdot \nabla u \mathrm{~d} x\right]_{S}^{T}+\int_{S}^{T} \phi^{\prime}(t) \int_{\Omega} u^{\prime} h \cdot \nabla u \mathrm{~d} x \mathrm{~d} t \\
& +\int_{S}^{T} \phi(t) \int_{\Omega} h \cdot \nabla u f \mathrm{~d} x \mathrm{~d} t \\
& -\int_{S}^{T} \phi(t) \int_{\Omega \cap Q_{1}}\left[\frac{1}{2} \operatorname{divh}\left(\left|u^{\prime}\right|^{2}-|\nabla u|^{2}\right)+\sum_{i, k} \frac{\partial u}{\partial x_{i}} \frac{\partial u}{\partial x_{k}} \frac{\partial h_{k}}{\partial x_{i}}\right] \mathrm{d} x \mathrm{~d} t .
\end{aligned}
$$

Using the second multiplier $\frac{N-1}{2} \phi(t) u$ for (5.4):

$$
\frac{N-1}{2} \int_{S}^{T} \phi(t) \int_{\Omega} u\left(u^{\prime \prime}-\Delta u-f\right) \mathrm{d} x \mathrm{~d} t=0,
$$

yields that

$$
\begin{aligned}
\frac{N-1}{2} \int_{S}^{T} \phi(t) \int_{\Omega}\left(|\nabla u|^{2}-\left|u^{\prime}\right|^{2}\right) \mathrm{d} x \mathrm{~d} t= & -\frac{N-1}{2}\left[\phi(t) \int_{\Omega} u^{\prime} u \mathrm{~d} x\right]_{S}^{T}+\frac{(N-1)}{2} \int_{S}^{T} \phi^{\prime}(t) \int_{\Omega} u^{\prime} u \mathrm{~d} x \mathrm{~d} t \\
& +\frac{N-1}{2} \int_{S}^{T} \phi(t) \int_{\Omega} u f \mathrm{~d} x \mathrm{~d} t .
\end{aligned}
$$

Adding (6.5) to (6.4) and using Cauchy-Schwarz and Poincaré's inequalities, we obtain for all $\delta_{1}>0$ that

$$
\begin{aligned}
\int_{S}^{T} \phi(t) e(t) \mathrm{d} t= & \int_{S}^{T} \phi(t) \int_{\Omega} \frac{|\nabla u|^{2}+\left|u^{\prime}\right|^{2}}{2} \mathrm{~d} x \mathrm{~d} t \\
\leq & C \phi(S)[e(S)+e(T)]+C \int_{S}^{T}-\phi^{\prime}(t) e(t) \mathrm{d} t \\
& +\delta_{1} \int_{S}^{T} \phi(t) e(t) \mathrm{d} t+\frac{C}{\delta_{1}} \int_{S}^{T} \phi(t) \int_{\Omega}|f|^{2} \mathrm{~d} x \mathrm{~d} t \\
& +C \int_{S}^{T} \phi(t) \int_{\Omega \cap Q_{1}}\left(\left|u^{\prime}\right|^{2}+|\nabla u|^{2}\right) \mathrm{d} x \mathrm{~d} t .
\end{aligned}
$$

Compared to the desired estimate (5.5), the term concerning $|\nabla u|^{2}$ on the right hand of (6.6) is crucial. We just follow the techniques developed in [33] to deal with this term.

Since $\overline{\mathbb{R}^{N} \backslash Q_{2}} \cap \bar{Q}_{1}=\emptyset$, there exists a cut-off function $\xi \in C_{0}^{\infty}(\mathbb{R})$ such that

$$
0 \leq \xi \leq 1, \quad \xi=1 \quad \text { on } Q_{1}, \quad \xi=0 \quad \text { on } \mathbb{R}^{N} \backslash Q_{2} .
$$

Applying now the multiplier $\phi(t) \xi(x) u$ to (5.4) gives, after integration by parts, that

$$
\begin{aligned}
\int_{S}^{T} \phi(t) \int_{\Omega} \xi|\nabla u|^{2} \mathrm{~d} x \mathrm{~d} t= & \int_{S}^{T} \phi(t) \int_{\Omega}\left(\xi\left|u^{\prime}\right|^{2}+\frac{1}{2} u^{2} \Delta \xi\right) \mathrm{d} x \mathrm{~d} t \\
& +\int_{S}^{T} \phi^{\prime}(t) \int_{\Omega} \xi u u^{\prime} \mathrm{d} x \mathrm{~d} t-\left[\phi(t) \int_{\Omega} \xi u u^{\prime} \mathrm{d} x\right]_{S}^{T} \\
& +\int_{S}^{T} \phi(t) \int_{\Omega} \xi u f \mathrm{~d} x \mathrm{~d} t .
\end{aligned}
$$


Then it follows from the definition of $\xi$ that

$$
\begin{aligned}
\int_{S}^{T} \phi(t) \int_{\Omega \cap Q_{1}}|\nabla u|^{2} \mathrm{~d} x \mathrm{~d} t \leq & \int_{S}^{T} \phi(t) \int_{\Omega} \xi|\nabla u|^{2} \mathrm{~d} x \mathrm{~d} t \\
\leq & C \phi(S)[e(S)+e(T)]+C \int_{S}^{T}-\phi^{\prime}(t) e(t) \mathrm{d} t \\
& +C \int_{S}^{T} \phi(t) \int_{\Omega}|f|^{2} \mathrm{~d} x \mathrm{~d} t \\
& +C \int_{S}^{T} \phi(t) \int_{\Omega \cap Q_{2}}\left(\left|u^{\prime}\right|^{2}+|u|^{2}\right) \mathrm{d} x \mathrm{~d} t .
\end{aligned}
$$

Now it remains to estimate the term concerning $|u|^{2}$ in (6.8). Since $\overline{R^{N} \backslash \omega} \cap \bar{Q}_{2}=\emptyset$, there exists a function $\beta \in C_{0}^{\infty}(\mathbb{R})$ such that

$$
0 \leq \beta \leq 1, \quad \beta=1 \quad \text { on } Q_{2}, \quad \beta=0 \text { on } \mathbb{R}^{N} \backslash \omega .
$$

Fix the $t$ variable and consider the solution $z$ of the following elliptic problem in space:

$$
\left\{\begin{array}{l}
\Delta z=\beta(x) u, \quad \text { in } \Omega, \\
z=0, \quad \text { on } \Gamma .
\end{array}\right.
$$

Hence, $z$ and $z^{\prime}$ satisfy the following estimates

$$
\begin{aligned}
\|z\|_{L^{2}} & \leq C \int_{\Omega} \beta(x)|u|^{2} \mathrm{~d} x . \\
\left\|z^{\prime}\right\|_{L^{2}}^{2} & \leq C \int_{\Omega} \beta(x)\left|u^{\prime}\right|^{2} \mathrm{~d} x .
\end{aligned}
$$

Applying the multiplier $\phi(t) z$ to (5.4) gives, after integration by parts, that

$$
\begin{aligned}
\int_{S}^{T} \phi(t) \int_{\Omega} \beta(x)|u|^{2} \mathrm{~d} x \mathrm{~d} t= & {\left[\phi(t) \int_{\Omega} z u^{\prime} \mathrm{d} x\right]_{S}^{T}-\int_{S}^{T} \phi^{\prime}(t) \int_{\Omega} z u^{\prime} \mathrm{d} x \mathrm{~d} t } \\
& +\int_{S}^{T} \phi(t) \int_{\Omega}\left(-z^{\prime} u^{\prime}-z f\right) \mathrm{d} x \mathrm{~d} t .
\end{aligned}
$$

Hence, using the estimates (6.10)-(6.11) in the above relation, and noting the definition of $\beta$, we obtain for all $\delta_{2}>0$

$$
\begin{aligned}
\int_{S}^{T} \phi(t) \int_{\Omega \cap Q_{2}}|u|^{2} \mathrm{~d} x \mathrm{~d} t \leq & \int_{S}^{T} \phi(t) \int_{\Omega} \beta(x)|u|^{2} \mathrm{~d} x \mathrm{~d} t \\
\leq & C \phi(S)[e(S)+e(T)]+C \int_{S}^{T}-\phi^{\prime}(t) e(t) \mathrm{d} t \\
& +\delta_{2} \int_{S}^{T} \phi(t) e(t) \mathrm{d} t+\frac{C}{\delta_{2}} \int_{S}^{T} \phi(t) \int_{\omega}\left|u^{\prime}\right|^{2} \mathrm{~d} x \mathrm{~d} t \\
& +\frac{C}{\delta_{2}} \int_{S}^{T} \phi(t) \int_{\Omega}|f|^{2} \mathrm{~d} x \mathrm{~d} t .
\end{aligned}
$$

Inserting (6.8) and (6.12) in (6.6), then choosing finally $\delta_{1}$ and $\delta_{2}$ sufficiently small, we obtain (5.5) which ends the Proof of Lemma 5.2. 
Acknowledgements. The authors are thankful to the support of the ERC advanced Grant 266907 (CPDENL) and the hospitality of the Laboratoire Jacques-Louis Lions of Université Pierre et Marie Curie. Fatiha Alabau-Boussouira was supported by the LIASFMA for a visit at Fudan University during August 2014. Zhiqiang Wang was partially supported by the National Science Foundation of China (No. 11271082) and by the State Key Program of National Natural Science Foundation of China (No. 11331004).

\section{REFERENCES}

[1] F. Alabau, Stabilisation frontière indirecte de systèmes faiblement couplés. C. R. Acad. Sci. Paris Sér. I Math. 328 (1999) $1015-1020$.

[2] F. Alabau, Indirect boundary stabilization of weakly coupled systems. SIAM J. Control Optim. 41 (2002) 511-541.

[3] F. Alabau-Boussouira, Une formule générale pour le taux de décroissance des systèmes dissipatifs non linéaires. $C$. $R$. Acad. Sci. Paris Sér I Math. 338 (2004) 35-40.

[4] F. Alabau-Boussouira, Convexity and weighted integral inequalities for energy decay rates of nonlinear dissipative hyperbolic systems. Appl. Math. Optim. 51 (2005) 61-105.

[5] F. Alabau-Boussouira, Piecewise multiplier method and nonlinear integral inequality for Petrowsky equation with nonlinear dissipation. J. Evol. Equ. 6 (2006) 95-112.

[6] F. Alabau-Boussouira, Asymptotic behavior for Timoshenko beams subject to a single nonlinear feedback control. NoDEA 14 (2007) 643-669.

[7] F. Alabau-Boussouira, A unified approach via convexity for optimal energy decay rates of finite and infinite dimensional vibrating damped systems with applications to semi-discretized vibrating damped systems. J. Differ. Equ. 248 (2010) 14731517 .

[8] F. Alabau-Boussouira, New trends towards lower energy estimates and optimality for nonlinearly damped vibrating systems. J. Differ. Equ. 249 (2010) 1145-1178.

[9] F. Alabau-Boussouira, Strong lower energy estimates for nonlinearly damped Timoshenko beams and Petrowsky equations. NoDEA 18 (2011) 571-597.

[10] F. Alabau-Boussouira, On some recent advances on stabilization for hyperbolic equations. Vol. 2048 of Lect. Note Math. CIME Foundation Subseries Control of Partial Differential Equations. Springer Verlag (2012) 101.

[11] F. Alabau-Boussouira and K. Ammari, Sharp energy estimates for nonlinearly locally damped PDEs via observability for the associated undamped system. J. Funct. Anal. 260 (2011) 2424-2450.

[12] F. Alabau-Boussouira and M. Léautaud, Indirect stabilization of locally coupled wave-type systems. ESAIM: COCV 18 (2012) $548-582$.

[13] F. Alabau-Boussouira and M. Léautaud, Indirect controllability of locally coupled wave-type systems and applications. $J$. Math. Pures Appl. 99 (2013) 544-576.

[14] F. Alabau, P. Cannarsa and V. Komornik, Indirect internal damping of coupled systems. J. Evol. Equ. 2 (2002) 127-150.

[15] F. Alabau-Boussouira, P. Cannarsa and R. Guglielmi, Indirect stabilization of weakly coupled systems with hybrid boundary conditions. Math. Control Relat. Fields 1 (2011) 413-436.

[16] F. Alabau-Boussouira, Y. Privat and E. Trélat, Nonlinear damped partial differential equations and their uniform discretizations. Preprint arXiv:1506.04163 (2015).

[17] C. Bardos, G. Lebeau and J. Rauch, Sharp sufficient conditions for the observation, control and stabilization of waves from the boundary. SIAM J. Control Optim. 30 (1992) 1024-1065.

[18] M. Bellassoued, Rate of decay of solution of the wave equation with arbitrary localized nonlinear damping. J. Differ. Equ. 211 (2005) 303-332.

[19] G. Chen, A note on boundary stabilization of the wave equation. SIAM J. Control Optim. 19 (1981) $106-113$.

[20] Y. Cui and Z. Wang, Asymptotic stability of wave equations coupled by velocities. Math. Control Relat. Fields 6 (2016) 429-446.

[21] B. Dehman, G. Lebeau and E. Zuazua, Stabilization and control for the subcritical semilinear wave equation. Annales Scientifiques de l'École Normale Supérieure 36 (2003) 525-551.

[22] X. Fu, Longtime behavior of the hyperbolic equations with an arbitrary internal damping. Z. Angew. Math. Phys. 62 (2011) $667-680$.

[23] X. Fu, Sharp decay rates for the weakly coupled hyperbolic system with one internal damping. SIAM J. Control Optim. 50 (2012) 1643-1660.

[24] R. Joly and C. Laurent, Stabilization for the semilinear wave equation with geometric control condition. Ann. PDE 6 (2012) $1089-1119$

[25] J.U. Kim and Y. Renardy, Boundary control of the Timoshenko beam. SIAM J. Control Optim. 25 (1987) $1417-1429$.

[26] V. Komornik, Exact controllability and stabilization: The Multiplier Method. Vol. 36 of Collection RMA. Masson-John Wiley, Paris-Chicester (1994).

[27] I. Lasiecka and D. Tataru, Uniform boundary stabilization of semilinear wave equation with nonlinear boundary damping. Differ. Integral Equ. 8 (1993) 507-533.

[28] G. Lebeau, Équation des ondes amorties, Algebraic and geometric methods in mathematical physics (Kaciveli, 1993). Math. Phys. Study. Kluwer Acad. Publ., Dordrecht (1996) 73-109. 
[29] G. Lebeau and E. Zuazua, Decay rates for the three-dimensional linear system of thermoelasticity. Arch. Ration Mech. Anal. 148 (1999) 179-231.

[30] J.-L. Lions, Contrôlabilité exacte et stabilisation de systèmes distributés. Vol. 1 of Collection RMA. Masson, Paris (1988).

[31] K. Liu, Locally distributed control and damping for the conservative systems. SIAM J. Control Optim. 35 (1997) 1574-1590.

[32] W.-J. Liu and E. Zuazua, Decay rates for dissipative wave equations. Ricerche di Matematica 48 (1999) 61-75.

[33] P. Martinez, A new method to obtain decay rate estimates for dissipative systems with localized damping. Rev. Mat. Complut. 12 (1999) 251-283.

[34] P. Martinez, A new method to obtain decay rate estimates for dissipative systems. ESAIM: COCV 4 (1999) 419-444.

[35] P. Martinez and J. Vancostenoble, Exponential stability for the wave equation with weak nonmonotone damping. Portugal. Math. 57 (2000) 285-310.

[36] M. Nakao, Decay of solutions of the wave equation with a local nonlinear dissipation. Math. Ann. 305 (1996) $403-417$.

[37] A. Soufyane, Stabilisation de la poutre de Timoshenko. C. R. Acad. Sci. Paris Sér I Math. 328 (1999) 731-734.

[38] J. Vancostenoble, Optimalité d'estimation d'énergie pour une équation des ondes amortie. C. R. Acad. Sci. Paris série I 328 (1999) 777-782.

[39] J. Vancostenoble and P. Martinez, Optimality of energy estimates for the wave equation with nonlinear boundary velocity feedbacks. SIAM J. Control Optim. 39 (2000) 776-797.

[40] E. Zuazua, Uniform stabilization of the wave equation by nonlinear feedbacks. SIAM J. Control Optim. 28 (1989) $265-268$.

[41] E. Zuazua, Exponential decay for the semilinear wave equation with locally distributed damping. Commun. Part. Differ. Equ. 15 (1990) 205-235. 